\title{
Unidirectional monosynaptic connections from auditory areas to the primary visual cortex in the marmoset monkey
}

\author{
Piotr Majka, ${ }^{1,2} \cdot$ Marcello G. P. Rosa ${ }^{2,3} \cdot$ Shi Bai ${ }^{2,3} \cdot$ Jonathan M. Chan ${ }^{3} \cdot$ Bing-Xing Huo $^{4,5} \cdot$ Natalia Jermakow $^{1}$. \\ Meng K. Lin ${ }^{4}$ - Yeonsook S. Takahashi ${ }^{4}$. Ianina H. Wolkowicz ${ }^{3}$ Katrina H. Worthy ${ }^{3} \cdot$ Ramesh Rajan $^{2,3}$. \\ David H. Reser ${ }^{6}$. Daniel K. Wójcik ${ }^{1}$ (D) Hideyuki Okano ${ }^{4,7} \cdot$ Partha P. Mitra $^{2,4,5}$
}

Received: 2 July 2018 / Accepted: 27 September 2018 / Published online: 4 October 2018

(c) The Author(s) 2018

\begin{abstract}
Until the late twentieth century, it was believed that different sensory modalities were processed by largely independent pathways in the primate cortex, with cross-modal integration only occurring in specialized polysensory areas. This model was challenged by the finding that the peripheral representation of the primary visual cortex (V1) receives monosynaptic connections from areas of the auditory cortex in the macaque. However, auditory projections to $\mathrm{V} 1$ have not been reported in other primates. We investigated the existence of direct interconnections between V1 and auditory areas in the marmoset, a New World monkey. Labelled neurons in auditory cortex were observed following 4 out of 10 retrograde tracer injections involving V1. These projections to V1 originated in the caudal subdivisions of auditory cortex (primary auditory cortex, caudal belt and parabelt areas), and targeted parts of V1 that represent parafoveal and peripheral vision. Injections near the representation of the vertical meridian of the visual field labelled few or no cells in auditory cortex. We also placed 8 retrograde tracer injections involving core, belt and parabelt auditory areas, none of which revealed direct projections from $\mathrm{V} 1$. These results confirm the existence of a direct, nonreciprocal projection from auditory areas to V1 in a different primate species, which has evolved separately from the macaque for over 30 million years. The essential similarity of these observations between marmoset and macaque indicate that early-stage audiovisual integration is a shared characteristic of primate sensory processing.
\end{abstract}

Keywords Striate cortex $\cdot$ Auditory cortex $\cdot$ Connections $\cdot$ Primate $\cdot$ Audiovisual integration

Piotr Majka and Marcello G. P. Rosa contributed equally to this study, and are listed here alphabetically as equal first authors.

Marcello G. P. Rosa

marcello.rosa@monash.edu

$\triangle$ Partha P. Mitra

parthaxmitra@gmail.com

1 Laboratory of Neuroinformatics, Nencki Institute of Experimental Biology of Polish Academy of Sciences, 02-093 Warsaw, Poland

2 Monash University Node, Australian Research Council, Centre of Excellence for Integrative Brain Function, Clayton, VIC 3800, Australia

3 Biomedicine Discovery Institute and Department of Physiology, Monash University, Clayton, VIC 3800, Australia
4 Laboratory for Marmoset Neural Architecture, RIKEN Center for Brain Science, Saitama 351-0106, Japan

5 Cold Spring Harbor Laboratory, Cold Spring Harbor, NY 11724, USA

6 School of Rural Health, Monash University, Churchill, VIC 3842, Australia

7 Department of Physiology, Keio University School of Medicine, Tokyo 160-8582, Japan 


\section{Introduction}

The integration of visual and auditory information is an important process in daily life. Congruent stimulation can improve the detectability of sensory targets that are weak or masked by noise, especially in situations involving low signal to noise ratios (Witten and Knudsen 2005; Ross et al. 2006). The information from each modality appears to be weighted according to its estimated reliability: in audiovisual interactions vision tends to dominate spatial aspects of the combined percept, while audition dominates temporal aspects (Witten and Knudsen 2005; Burr and Alais 2006). Task difficulty also influences the likelihood of audiovisual fusion (Battaglia et al. 2003; Alsius et al. 2005; Wang et al. 2008).

The fact that information from one sensory modality can influence what we perceive through another modality implies the existence of polysensory neurons, which respond to more than one type of sensory input, or whose responses to one modality are enhanced or inhibited by concurrent stimulation of another modality. In the primate cerebral cortex, it has been known since the 1980s that overt polysensory responses occur in regions of the superior temporal, parietal and frontal cortices (see Beauchamp 2005; Macaluso and Driver 2005; van Atteveldt et al. 2014; Yau et al. 2015 for reviews). Yet, subtler cross-modal effects have been detected in regions originally thought to be strictly unimodal, including in visual and auditory areas (e.g. Ghazanfar and Schroeder 2006; Wang et al. 2008; Kayser et al. 2009).

In the classical view, the confluence of visual and auditory inputs only occurs in high-order, polysensory association cortices, after many hierarchical steps (Jones and Powell 1970; Felleman and Van Essen 1991). This anatomical model reflects the original physiological evidence that polysensory neurons are restricted to specialized association cortices (e.g. Benevento et al. 1977; Bruce et al. 1981). As these areas lack direct connections with primary and secondary sensory cortices, the model implies that low-level crossmodal physiological effects depend on chains of "feedback" connections. However, others have challenged this hierarchical model, highlighting the potential role of small projection pathways in providing "shortcuts" between early-stage unimodal processing areas (Schroeder et al. 2003; Cappe and Barone 2005; Palmer and Rosa 2006a; Smiley et al. 2007; Smiley and Falchier 2009; Falchier et al. 2010). One of the earliest, and most powerful findings underlying the current model of early-stage integration of visual and auditory information in the primate cortex refers to the existence of direct (monosynaptic) connections from auditory areas to the primary visual area (V1) in macaques. This projection (Falchier et al. 2002; Rockland and Ojima 2003) is reported to originate from caudal auditory areas, as well as adjacent polysensory cortex, and to target primarily the peripheral representation of V1.

Here, we explored the existence of direct interconnections between auditory areas and V1 in the marmoset monkey (Callithrix jacchus), a New World monkey which is attracting increasing interest as a model species for studies of neural systems that are highly developed or specialized in the primate brain (Solomon and Rosa 2014; Mitchell and Leopold 2015; Majka et al. 2016; Miller et al. 2016; Eliades and Miller 2017; Hagan et al. 2017; Oikonomidis et al. 2017; Okano et al. 2016; Okano and Kishi 2017; Silva 2017). Previous work in this species has indicated that sparse projections from auditory cortex target the middle temporal visual area (MT; Palmer and Rosa 2006a), but studies of V1 connections have not reported afferents from the auditory cortex (Rosa and Tweedale 2000; Lyon and Kaas 2001). Here, we report that the peripheral representation of $\mathrm{V} 1$ receives a sparse projection from the caudal auditory cortex and adjacent polysensory areas, which is not reciprocated by a V1 projection to auditory cortex. The similarity of observations between marmoset and macaque, species that have evolved separately for over 30 million years, indicate that early-stage audiovisual integration is a shared characteristic of primate sensory processing.

\section{Materials and methods}

Experiments involved 11 adult marmoset monkeys ( Callithrix jacchus). Tracer injections were performed both at Monash University (cases CJ64, CJ75, CJ82, CJ122, CJ174, CJ178, CJ180 and CJ802) and the RIKEN Brain Science Institute (BSI, which was re-established as RIKEN Center for Brain Science [CBS] in April 2018); cases M820, M822 and M1146. Very similar methods were used on both locations (where applicable, differences are detailed below). Experiments in Australia conformed to the Australian Code of Practice for the Care and Use of Animals for Scientific Purposes, and were approved by the Monash University Animal Experimentation Ethics Committee, and those in Japan were approved by the Institutional Animal Care and Use Committee at RIKEN, and conducted in accordance with the Guidelines for Conducting Animal Experiments at RIKEN BSI. Participation of Australian Researchers in surgeries conducted at the RIKEN BSI was authorized by a field license obtained from the Monash University Animal Experimentation Ethics Committee. Each of the animals received multiple injections of fluorescent tracers, which were aimed at specific locations in the cortex using stereotaxic coordinates (Paxinos et al. 2012). The exact areas involved in each injection were subsequently determined based on histological examination (Table 1). A full list of the abbreviations of cortical areas that contained labelled 
Table 1 Animals and injections

\begin{tabular}{|c|c|c|c|c|c|c|c|c|c|c|}
\hline Animal & Age (months) & Sex & Weight (g) & $\begin{array}{l}\text { Hemi- } \\
\text { sphere } \\
\text { injected }\end{array}$ & $\begin{array}{l}\text { Survival } \\
\text { time } \\
\text { (days) }\end{array}$ & Tracer & Area(s) injected & Layers injected & $\begin{array}{l}\text { White mat- } \\
\text { ter involved }\end{array}$ & $\begin{array}{l}\text { Injection vol- } \\
\text { ume }\left(\mathrm{mm}^{3}\right)^{\mathrm{a}}\end{array}$ \\
\hline \multirow[t]{3}{*}{ CJ82 } & 28 & $\mathrm{~F}$ & 375 & $\mathrm{~L}$ & 15 & FE & V1 & $3-4$ & $\mathrm{~N}$ & 0.14 \\
\hline & & & & & & FR & V1 & $2-6$ & $\mathrm{~N}$ & 0.10 \\
\hline & & & & & & DY & $\mathrm{V} 2, \mathrm{~V} 1$ & $1-3$ & $\mathrm{~N}$ & 0.11 \\
\hline \multirow[t]{3}{*}{ CJ174 } & 30 & $\mathrm{~F}$ & 342 & $\mathrm{R}$ & 17 & CTB (g) & V1 & $1-3$ & $\mathrm{~N}$ & 0.11 \\
\hline & & & & & & CTB (r) & V1 & $1-3$ & $\mathrm{~N}$ & 0.03 \\
\hline & & & & & & FB & V1 & $1-6$ & $\mathrm{H}$ & 1.19 \\
\hline CJ178 & 26 & $\mathrm{~F}$ & 344 & $\mathrm{R}$ & 18 & FB & V1 & $1-6$ & $\mathrm{C}$ & 0.49 \\
\hline M820 & 83 & $\mathrm{~F}$ & 400 & $\mathrm{~L}$ & 30 & FB & V1 & $1-6$ & $\mathrm{H}$ & 0.63 \\
\hline M822 & 85 & $\mathrm{~F}$ & 355 & $\mathrm{~L}$ & 29 & FB & V1, V2 & $1-6$ & $\mathrm{~N}$ & 0.81 \\
\hline M1146 & 85 & $\mathrm{~F}$ & 394 & $\mathrm{~L}$ & 21 & FB & V1 & $1-3$ & $\mathrm{~N}$ & 0.05 \\
\hline \multirow[t]{2}{*}{ CJ64 } & 16 & M & 303 & $\mathrm{R}$ & 14 & FR & $\mathrm{CM}, \mathrm{A} 1$ & $1-5$ & $\mathrm{~N}$ & 0.17 \\
\hline & & & & & & $\mathrm{FE}$ & $\mathrm{AL}, \mathrm{ML}$ & $1-5$ & $\mathrm{~N}$ & 0.17 \\
\hline CJ75 & 16 & M & 356 & $\mathrm{R}$ & 10 & FR & A1 & $1-6$ & $\mathrm{~N}$ & 0.12 \\
\hline \multirow[t]{2}{*}{ CJ122 } & 34 & M & 318 & $\mathrm{R}$ & 14 & DY & RT, AL & $1-6$ & $\mathrm{H}$ & 0.17 \\
\hline & & & & & & FR & $\mathrm{CPB}, \mathrm{AL}$ & $1-4$ & $\mathrm{~N}$ & 0.05 \\
\hline \multirow[t]{2}{*}{ CJ180 } & 32 & M & 374 & $\mathrm{R}$ & 11 & FB & RT, AL, RTL & $1-6$ & $\mathrm{C}$ & 0.99 \\
\hline & & & & & & CTB $(\mathrm{g})$ & ML, CPB & $1-6$ & $\mathrm{~N}$ & 0.11 \\
\hline CJ802 & 43 & $\mathrm{M}$ & 352 & $\mathrm{R}$ & 22 & CTB $(\mathrm{g})$ & ML, CL, CPB & $1-6$ & $\mathrm{~N}$ & 0.17 \\
\hline
\end{tabular}

$L$ left, $R$ right, $C T B(g)$ cholera toxin subunit B, conjugated with Alexa 488, $C T B(r)$ cholera toxin subunit B, conjugated with Alexa $594, D Y$ diamidino yellow dihydrochloride, $2 \%$ in $\mathrm{dH}_{2} \mathrm{O}, F B$ fast blue, $2 \%$ in $\mathrm{dH}_{2} \mathrm{O}, F E$ fluoroemerald (fluorescein dextran-amine, $\mathrm{MW} 10000$ ), $10 \%$ in $\mathrm{dH}_{2} \mathrm{O}, F R$ fluororuby (tetramethylrhodamine dextran-amine MW 10000 ), $10 \%$ in $\mathrm{dH}_{2} \mathrm{O}, C$ core, $H$ halo, $N$ no involvement

${ }^{a}$ Injection site volume estimated across multiple histological sections, using the criteria proposed by Condé (1987) and Schmued et al. (1990). Measurements uncorrected for shrinkage due to histological processing

neurons following the present injections is presented in Table 2. In all figures the sections and maps are illustrated using the convention appropriate for a left hemisphere, to facilitate comparisons between cases and with earlier publications from our laboratories. The actual hemisphere in which the injections were placed is given for each case in Table 1. The extents of diamidino yellow (DY) and fast blue (FB) injection sites were estimated according to the criteria defined by Condé (1987). Other injections were estimated as the volume of cortex containing fluorescent dye in the extracellular space (which is limited to the neighbourhood of the needle track; Schmued et al. 1990).

\section{Surgeries}

The surgical procedures have been described in detail previously (Reser et al. 2013; Burman et al. 2010, 2014a, b). Intramuscular (i.m.) injections of atropine $(0.2 \mathrm{mg} / \mathrm{kg})$ and diazepam $(2 \mathrm{mg} / \mathrm{kg})$ were administered as pre-medication before each animal was anaesthetized with alfaxalone $(10 \mathrm{mg} / \mathrm{kg}$, i.m. $) 30 \mathrm{~min}$ later. Dexamethasone $(0.3 \mathrm{mg} / \mathrm{kg}$, i.m.) and amoxicillin $(50 \mathrm{mg} / \mathrm{kg}$, i.m.) were also administered prior to positioning the animals in a stereotaxic frame. Body temperature, heart rate, and blood oxygenation (PO2) were continuously monitored during surgery, and when necessary, supplemental doses of anaesthetic were administered to maintain areflexia. Incisions of the dura mater were made over the intended injection sites to limit exposure of the brain's surface. Tracer injections were placed in the same hemisphere in each animal.

Six types of fluorescent tracers were used (Table 1): fluororuby (FR; dextran-conjugated tetramethylrhodamine, molecular weight $10,000,15 \%$ ), fluoroemerald (FE; dextranconjugated fluorescein, molecular weight 10,000,15\%), fast blue (FB), diamidino yellow (DY) and CTB [cholera toxin subunit B, conjugated with either Alexa 488 (CTBg) or Alexa $594(\mathrm{CTBr})]$. The dextran tracers resulted in bidirectional transport, but only retrograde labelling is reported here. In most cases, the tracers were injected using $25 \mu \mathrm{l}$ constant rate microsyringes (Hamilton, Reno, NV) fitted with a fine glass micropipette tip. In cases M820, M822 and M1146, the injections used a Nanoject II injector (Drummond Scientific, Broomall, PA, USA), also fitted with a micropipette, with dosage controlled by Micro4 microsyringe pump controller (World Precision Instruments, Sarasota, FL, USA). Each tracer was injected over 15-20 min, with small deposits of tracer made at different depths. Following the last deposit, the pipette was left in place for 
Table 2 Abbreviations of names of cortical areas

1/2: areas 1 and 2 of somatosensory cortex

6DR: cytoarchitectural area 6 of cortex, dorsorostral part

$8 \mathrm{aV}$ : cytoarchitectural area $8 \mathrm{a}$ of cortex, ventral part

8b: cytoarchitectural area $8 \mathrm{~b}$ of cortex

10: cytoarchitectural area 10 of cortex (frontopolar cortex)

11: cytoarchitectural area 11 of cortex

12L: cytoarchitectural area 12 of cortex (area 47), lateral part

12M: cytoarchitectural area 12 of cortex (area 47), medial part

13L: cytoarchitectural area 13 of cortex, lateral part

13M: cytoarchitectural area 13 of cortex, medial part

19M: cytoarchitectural area 19 of visual cortex, medial part

23a: cytoarchitectural area 23a of cortex

$23 \mathrm{~V}$ : cytoarchitectural area 23 of cortex, ventral part

32: cytoarchitectural area 32 of cortex

36: cytoarchitectural area 36 of cortex

45: cytoarchitectural area 45 of cortex

A1: primary auditory area

AL: anterolateral auditory area

CL: caudolateral auditory area

CM: caudomedial auditory area

CPB: caudal parabelt auditory area

DM: dorsomedial visual area (V6)

FST: fundus of superior temporal sulcus area

GI: granular insular cortex

LIP: lateral intraparietal area

ML: middle lateral auditory area

MST: medial superior temporal area

MT: middle temporal area (V5)

MTc: middle temporal crescent area (V4T)

PaIM: parainsular cortex, medial part

PFG: cytoarchitectural area PFG

PG: cytoarchitectural area PG

$\mathrm{PGa} / \mathrm{IPa}$ : cytoarchitectural areas PGa and IPa

PR: parietal rostral area (somatosensory)

ProSt: area prostriata

PV: parietal ventral area (somatosensory)

$\mathrm{R}$ : rostral auditory area

ReI: retroinsular area

$\mathrm{RM}$ : rostromedial auditory area

RPB: rostral parabelt auditory area

RT: rostrotemporal auditory area

RTL: rostrotemporal lateral auditory area

S2: secondary somatosensory cortex

TE1: cytoarchitectural area TE, part 1

TE3: cytoarchitectural area TE, part 3

TEO: cytoarchitectural area TE, occipital transition part

TF: cytoarchitectural area TF

TFO: cytoarchitectural area TF, occipital transition part

$\mathrm{TH}$ : cytoarchitectural area TH
Table 2 (continued)

TL: cytoarchitectural area TL

TLO: cytoarchitectural area TL, occipital transition part

TPO: temporo-parieto-occipital association area (superior temporal polysensory cortex, STP)

TPOc: temporo-parieto-occipital association area, caudal subdivision

TPPro: temporopolar proisocortex

TPt: temporoparietal transitional area

V1: primary visual cortex

V2: visual area 2

V3: visual area 3 (ventrolateral posterior area, VLP)

V3a: visual area 3a (dorsoanterior area, DA)

V4: visual area 4 (ventrolateral anterior area, VLA)

V6a: visual area 6a (posterior parietal medial area)

VIP: ventral intraparietal area

3-5 min to minimize tracer reflux. Estimates of injection extent for each case, drawn under microscopic examination, are listed in Table 1. After the injections, the surface of the brain was covered with moistened ophthalmic film, over which the dural flaps were carefully arranged. The excised bone fragment was repositioned and secured in place with dental acrylic, and the wound closed in anatomical layers. Postoperative injectable analgesics were administered immediately after the animal exhibited spontaneous movements (Temgesic $0.01 \mathrm{mg} / \mathrm{kg}$, i.m., and Carprofen $4 \mathrm{mg} / \mathrm{kg}$, s.c.), followed by oral Metacam $(0.05 \mathrm{mg} / \mathrm{kg})$ for 3 consecutive days.

\section{Tissue processing and data analysis (experiments performed at Monash University)}

Survival times were between 11 and 22 days (Table 1), after which the animals were anesthetized with alfaxalone $(10 \mathrm{mg} /$ $\mathrm{ml}$ i.m.) and, following loss of consciousness, administered an overdose of sodium pentabarbitone $(100 \mathrm{mg} / \mathrm{kg}$, i.v. $)$. They were then immediately perfused through the heart with 11 of heparinized saline, followed by 11 of $4 \%$ paraformaldehyde (PFA) in $0.1 \mathrm{M}$ phosphate buffered saline (PBS; $\mathrm{pH}$ 7.4). The brains were post-fixed in the same medium for at least $24 \mathrm{~h}$, and then immersed in buffered paraformaldehyde with increasing concentrations of sucrose (10-30\%). They were then sectioned ( $40 \mu \mathrm{m}$ thickness) in the coronal plane, using a cryostat. One section in five was mounted unstained for examination of fluorescent tracers, and coverslipped after quick dehydration $(2 \times 100 \%$ ethanol $)$ and defatting $(2 \times$ xylene $)$. Adjacent sections were stained for Nissl substance, cytochrome oxidase, and myelin, following standard protocols (Gallyas 1979; Wong-Riley 1979). The remaining section in each series was stored in cryoprotectant solution in a freezer, to be used as a backup in case of unsatisfactory staining or damage during processing of the 
histological sections. Hence, the spacing between adjacent sections in each series was $200 \mu \mathrm{m}$ in these cases.

Sections were examined using a Zeiss Axioplan 2 epifluorescence microscope. Labelled neurons were identified using $\times 10$ or $\times 20$ dry objectives, and their locations within the cortex and subcortical structures were mapped using a digitizing system (MD Plot3, Accustage) attached to the microscope. To minimize the problem of overestimating the number of neurons due to the inclusion of cytoplasmic fragments, labelled cells were accepted as valid only if a nucleus could be discerned. This was straightforward in the case of DY, since this tracer only labels the neuron's nucleus (Keizer et al. 1983). In the case of tracers that label the cytoplasm (FB, FE, FR, CTBg and CTBr), the nucleus was discerned as a profile in the centre of a brightly lit, well-defined cell body, which in the vast majority of cases had an unmistakable pyramidal morphology.

To allow assessment of the areas in which injection sites and labelled neurons were located, each brain was reconstructed in 3 dimensions. Nissl-stained sections were scanned using Aperio Scanscope AT Turbo (Leica Biosystems) at $20 \times$ magnification, providing a resolution of $0.50 \mu \mathrm{m} / \mathrm{pixel}$. Within each coronal section, we identified the cortical areas proposed by Paxinos et al. (2012), based primarily on cytoarchitectural features observable in Nisslstained sections, but also using differences in myeloarchitecture and intensity of cytochrome staining as additional criteria to refine the delineations (e.g. Rosa et al. 2005, 2009; Palmer and Rosa 2006a, b; Burman and Rosa 2009; Burman et al. 2006, 2010, 2014a, b, 2015). This was followed by computerized 3-dimensional reconstruction of the entire hemisphere, and registration of each case to a template brain (Majka et al. 2016), to allow comparison between results from different cases. The registration procedure was supported by manually-drawn cyto-, myelo- and chemoarchitectonic boundaries, thus ensuring that the spatial relationships between the injection sites, labelled neurons, and histological boundaries were adequately maintained.

\section{Tissue processing and data analysis (experiments performed at RIKEN)}

The concurrent use of virus-based anterograde tracers (results to be reported separately) demanded longer survival times (21-30 days; Table 1) for these animals. At the end of the survival time they were anesthetized with a combination of ketamine $(10 \mathrm{mg} / \mathrm{kg})$ and diazepam $(2 \mathrm{mg} / \mathrm{kg})$. Following the loss of consciousness, they were administered a lethal injection of pentobarbital $(80 \mathrm{mg} / \mathrm{kg})$ and perfused through the heart with $500 \mathrm{ml}$ of heparinised PBS (pH 7.2) and $500 \mathrm{ml}$ of $4 \%$ PFA in $0.1 \mathrm{M}$ phosphate buffer (PB). The brains were removed from the skull, stored in the same fixation medium overnight, and then transferred to a $0.1 \mathrm{M}$
PB solution. A post-mortem MRI (data not used in the present study) was obtained at this stage, with Diffusion Tensor Imaging (DTI) and $300 \mu \mathrm{m}$ T2-weighted images (T2WI). Once the ex-vivo MRI was finished, the brains were transferred into increasing concentrations of sucrose in $0.1 \mathrm{M}$ PB (10\% for $24 \mathrm{~h}$, then $30 \%$ until sunk), and then embedded in Neg50 frozen section medium (Richard Allen Scientific, Waltham, MA) using a custom made polylactic acid base, a 3d-printed brain mould, and 2-methylbutane chilled with dry ice. Following embedding the brains were stored at $-80{ }^{\circ} \mathrm{C}$ until sectioning.

Each brain was sectioned at $20 \mu \mathrm{m}$ thickness in the coronal plane using a cryostat and a tape transfer technique (Pinskiy et al. 2015), which minimises distortions in section morphology during mounting. Three consecutive series of sections were stained for Nissl substance, myelinated fibres (Gallyas 1979), and cholera toxin subunit B immunocytochemistry (Angelucci et al. 1996; results not reported here), whereas the fourth series was kept unstained for fluorescent tracer analysis. Thus, the spacing between consecutive sections in each series was $80 \mu \mathrm{m}$ in these cases. Following curing with UV light, the slides used for the analysis of fluorescence were placed in xylene and coverslipped. All slides were scanned using a Nanozoomer 2.0 HT (Hamamatsu, Japan) equipped with a $20 \times$ objective $(0.46 \mu \mathrm{m} /$ pixel in plane) at 12-bit depth. The Nissl and myelin series were scanned with bright field illumination and the fluorescence series with a tri-pass filter cube (FITC/TX-RED/DAPI) for excitation. All imaging data were processed with a highthroughput neurohistological pipeline (Lin et al. 2018), resulting in high resolution images (e.g. Fig. 5).

\section{Results}

Figure 1 illustrates the locations of the centres of the 18 tracer injections analysed in the present study. Of these, 10 were located in V1, and 8 in various auditory areas (Table 1). Although the present analysis is focused on the evidence for direct cross-modal (auditory-visual) cortico-cortical connections, each of the injections also resulted, as expected, in retrograde label that extended across multiple cortical and subcortical areas. The full pattern of corticocortical label obtained in 8 of the animals (experiments conducted at Monash University) can be visualised, section by section, in a freely accessible website (http://www.marmosetbrain.org), which includes online tools for quantification; here, it will be summarised using "unfolded" representations of the cortex obtained by computational registration of the areas in each brain to a common template (Majka et al. 2016), and only direct connections between visual and auditory areas will be discussed. Data from the other 3 animals (experiments 


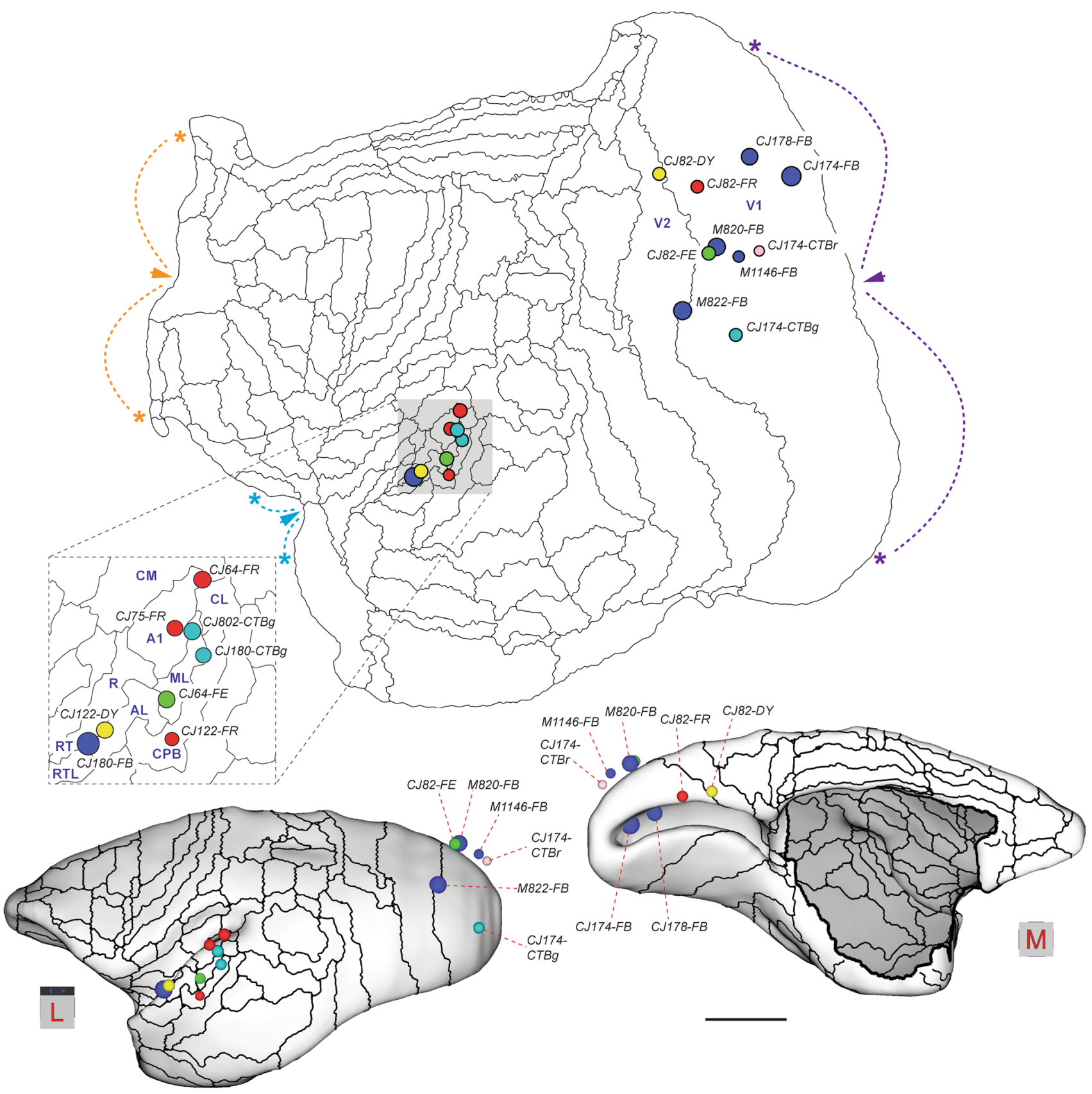

Fig. 1 Locations of 18 tracer injections analysed in the present study, plotted on a template of the marmoset cortex (reconstructed by Majka et al. 2016, from data published by; Paxinos et al. 2012). The template can be freely downloaded from http://www.marmosetbrain.org/ reference. Top: Unfolded reconstruction of the cortex (left hemisphere representation; rostral to the left, medial to the top) showing the location of each injection registered to the template. The colours of each circle represent the tracer used (blue-FB; yellow-DY; red-FR; green-FE; pink-CTBr; teal-CTBg), and their radius is proportional to that of a sphere with equal volume to the injection site reconstructed across histological sections (Table 1). The boundaries of cortical areas are indicated by thin lines. The auditory cortex (shaded in grey) is shown magnified in the insert. Arrowheads and asterisks indicate three points along the perimeter of the map where discontinuities were made to minimise distortions (fundus of the calcarine sulcus in purple, boundary between orbitofrontal cortex and medial frontal cortex in orange, piriform cortex in blue). For abbreviations, see Table 2. Bottom: 3-dimensional views of the marmoset brain from dorsolateral (left) and medial (right) perspectives, showing the locations of the injections in a reconstruction of the cortex at the level of its midthickness (for this reason, injections located in the supragranular layers appear to "float" above the cortex). Scale bar: $5 \mathrm{~mm}$ 
conducted at the RIKEN CBS) are hosted in http://marmo set.brainarchitecture.org.

\section{Projections from auditory areas to V1}

Our main observation is that the caudal auditory cortex sends a sparse projection to the portions of V1 located in the calcarine sulcus and midline. The most obvious label in auditory areas was observed in animal CJ174, following an injection of FB in the dorsal bank of the calcarine sulcus, with slight invasion of the ventral bank. This injection was centred in the lower quadrant representation near the horizontal meridian, at approximately $15-20^{\circ}$ eccentricity (Fritsches and Rosa 1996; Chaplin et al. 2013), but also included a portion of the upper quadrant representation (Fig. 2A). This resulted in widespread intrinsic label in V1, and extrinsic label in the peripheral representations of both dorsal and ventral visual cortex areas (Fig. 2A). There was no evidence of direct tracer spread to other areas (Fig. 2B, C). Figure 2D shows a summary view of the cortico-cortical connections revealed by this injection, which yielded labelled neurons in the various auditory and polysensory areas, and Fig. 3 illustrates examples of the locations of labelled neurons. V1-projecting neurons were found in the primary auditory area (A1; 22 neurons), the caudomedial (CM), caudolateral (CL) and middle lateral (ML) belt areas (30, 21 and 19 neurons, respectively), the caudal parabelt area (CPB; 7 neurons) and in the temporoparietal transition auditory association area (TPt; 15 neurons). There were also labelled neurons in putative polysensory areas (TPO and $\mathrm{PGa} / \mathrm{IPa}$ ). Despite the clearly defined labelled cells, the combined auditory projection accounted for a small proportion of the afferents to V1 (114 / 15,148, or $0.75 \%$ of the labelled cortico-cortical extrinsic connection neurons). Most (>90\%) of the labelled neurons were located in the infragranular layers, although in some sections supragranular neurons were also observed (Fig. 3).

Figure 4 illustrates data from a second animal (CJ178) with a FB injection in the calcarine sulcus. In this case, damage subsequent to the penetration of the injection syringe resulted in necrosis of the overlying optic radiations and parts of V2, which made impossible to ascertain the possibility of leakage (Fig. 4A). Nonetheless, the pattern of label in the cortex (Fig. 4B) was highly compatible with that observed in CJ174, although the total number of labelled neurons was much smaller (extrinsic cortico-cortical neurons in CJ178: 3,837). Based on comparison with Chaplin et al. (2013), this injection site was located further away from the representation of the horizontal meridian, in a region of $\mathrm{V} 1$ corresponding to $25-30^{\circ}$ eccentricity in the lower visual field. Label in auditory cortex was again sparse ( $0.23 \%$ of the extrinsic projections to V1), but originated in a subset of the same areas that formed projections in CJ174.
Figure 5 illustrates examples of FB-labelled neurons in auditory areas following the injection in case M820. The injection site was located in the representation of parafoveal vision in V1, close to the midline, and away from the representation of the vertical meridian (Figs. 1, 5A), as confirmed by the distribution of label in the lateral geniculate nucleus (Fig. 5B). Labelled neurons were located in the same areas that formed projections to V1 in cases CJ174 and CJ178 (e.g. Figs. 5C, 6). Quantification of these data confirmed the existence of a strong laminar bias in the projection, whereby each of the labelled cells was located in the infragranular layers and the fact that auditory projections form a small proportion of the extrinsic cortical afferents to V1 $(0.17 \%)$. A fourth case, M822, had a FB injection site at the V1/V2 border, at a more central location $\left(\sim 3^{\circ}\right.$ eccentricity). Only a few labelled neurons were observed in auditory areas $(n=6)$, all of which were located in the infragranular layers of caudal lateral belt cortex. These neurons formed $<0.1 \%$ of the extrinsic cortical projections to V1.

Figure 7 summarises the distribution of labelled cells in the auditory cortex in all cases reported in the present study. The cells in A1 tended to concentrate in the caudal part (representation of high-frequency sounds; Bendor and Wang 2008). Other concentrations of labelled neurons occurred in the caudal part of areas CM, ML, and CPB, and in area CL.

\section{Injections that revealed no evidence of projections from the auditory cortex}

Six other injections showed no evidence of labelled neurons in auditory areas, despite clear evidence of long-range tracer transport over long distances (e.g, to the frontal lobe). Summary maps of the pattern of label in five of these cases are shown in Fig. 8 (the results from animal M1146 could not be registered to the template, preventing display in flat map format). Four of the injections that resulted in no label in auditory cortex (CJ174-CTBr, CJ174-CTBg, CJ82-FE and $\mathrm{M} 1146)$ were in parts of V1 representing central vision $\left(<5^{\circ}\right.$ eccentricity), and two (CJ82-FR and CJ82-DY) were located at the representation of the peripheral lower vertical meridian of the visual field $\left(\sim 10^{\circ}\right.$ and $20^{\circ}$ eccentricity, respectively) in V1 (CJ82-FR) or V2, with small involvement of V1 (CJ82-DY). With one exception (CJ82-FR) these injections were all restricted to the supragranular layers; hence they may not have labelled the entire complement of afferents to this area.

\section{Injections in auditory cortex}

To explore the possibility of a reciprocal projection from auditory areas to $\mathrm{V} 1$, we have studied the connections revealed by injections in various auditory areas. As detailed 

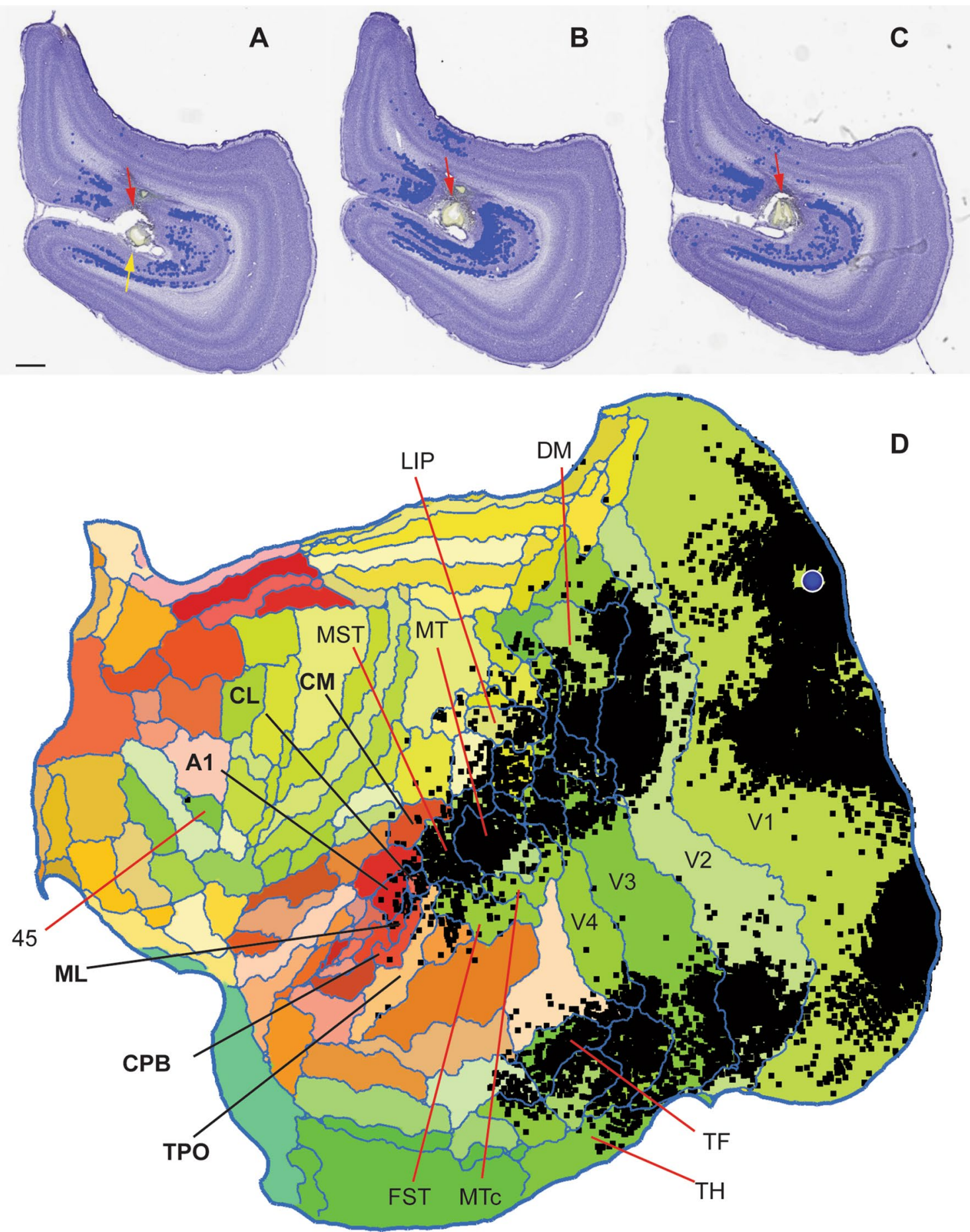

Fig. 2 Summary of results of a FB injection in animal CJ174. A-C Extent of the injection site, in coronal sections through the right hemisphere of a marmoset brain [anteroposterior (AP) levels $-8.6 \mathrm{~mm}$ to $-9.0 \mathrm{~mm}$; section A is the most rostral; lateral to the right]. Scale bar: $1 \mathrm{~mm}$. The red arrows point to the main injection site in the dorsal bank of the calcarine sulcus, and the yellow arrow points to the slight invasion of the ventral bank. At these levels, the entire section is formed by V1. Blue symbols represent labelled neurons forming intrinsic connections within V1. D Unfolded reconstruction of the cortex showing the locations of neurons labelled by the tracer injec- tion (black squares) following registration to the template. The injection site is indicated by the blue circle. In this and following figures, "flat" maps are oriented in the convention adopted for the Marmoset Brain Architecture Project (http://marmosetbrain.org), which represent a left hemisphere (rostral to the left, medial to the top), to facilitate comparison across cases (e.g. Paxinos et al. 2012; Majka et al. 2016), and the auditory cortex corresponds to a cluster of areas indicated in tones of red and orange. For the actual hemisphere injected, see Table 1. For orientation, the locations of several cortical areas are indicated 


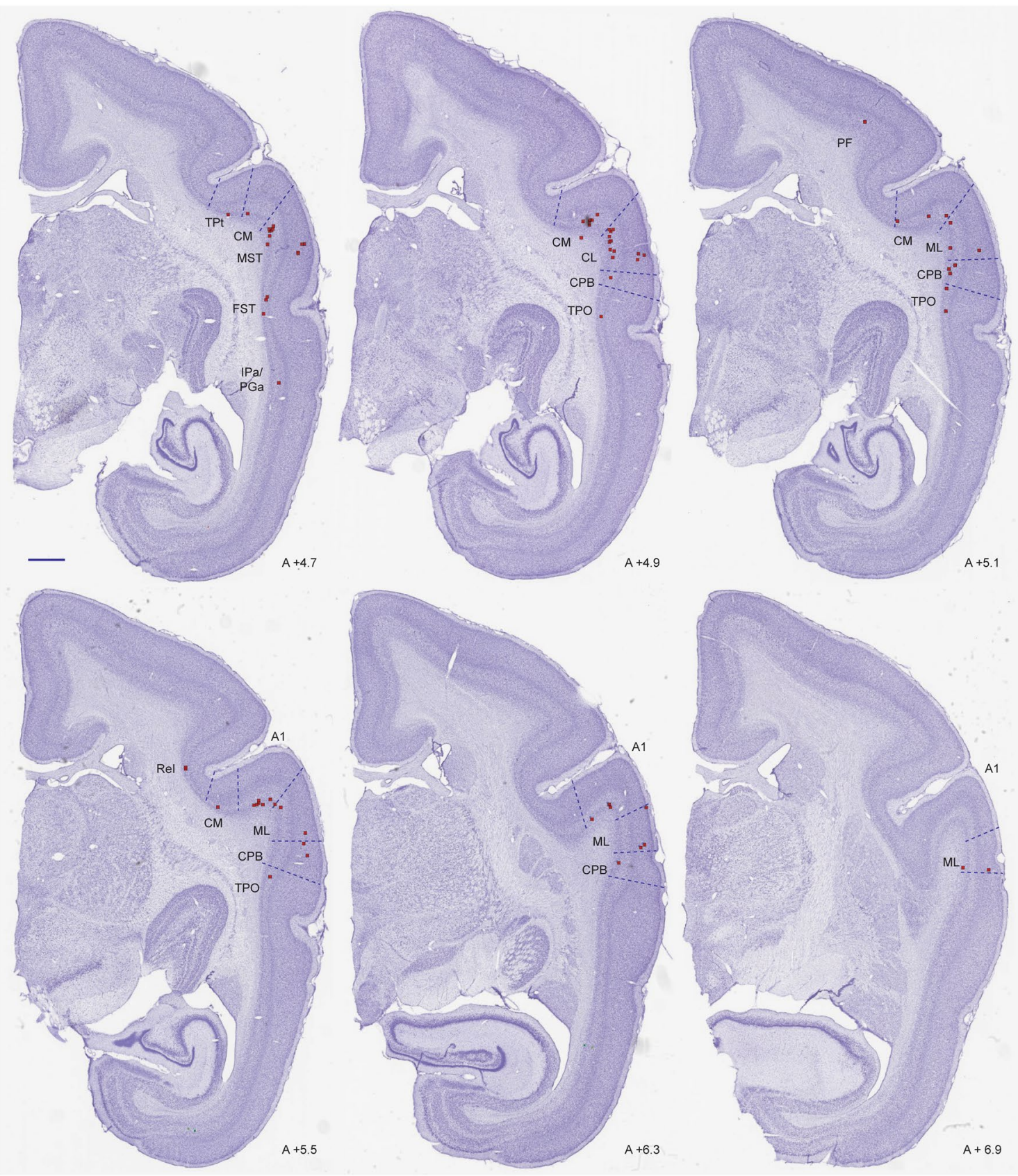

Fig. 3 Coronal sections through auditory cortex in case CJ174-FB, showing the locations of some of the neurons labelled by the V1 injection shown in Fig. 2 (red squares). The AP level of each section is indicated. Scale bar (top left): $1 \mathrm{~mm}$

below, despite label in a few other visual areas, none of these revealed direct projections from V1 (Figs. 9, 10).

Animal CJ75 had a FR tracer injection that encompassed all layers of the lateral part of A1. As shown in Fig. 9A, this injection resulted in a very concentrated pattern of label, mostly involving other auditory areas. The only possible visual projections originated in the most rostral part of the medial superior temporal (MST) and fundus of superior temporal (FST) areas, which accounted for $6.04 \%$ of the extrinsic afferents. As discussed below, the rostral sector of MST was the most commonly labelled region among putative visual areas, suggesting that this area, as defined in Paxinos et al. (2012), may be functionally heterogeneous. Putative polysensory projections were observed from the retroinsular (ReI) and temporoparietal transition (TPt) areas.

Two other injections involved the auditory core areas. In case CJ122-DY (Fig. 9B), the injection site was near the border of the rostral (R) and rostrotemporal (RT) areas, with 


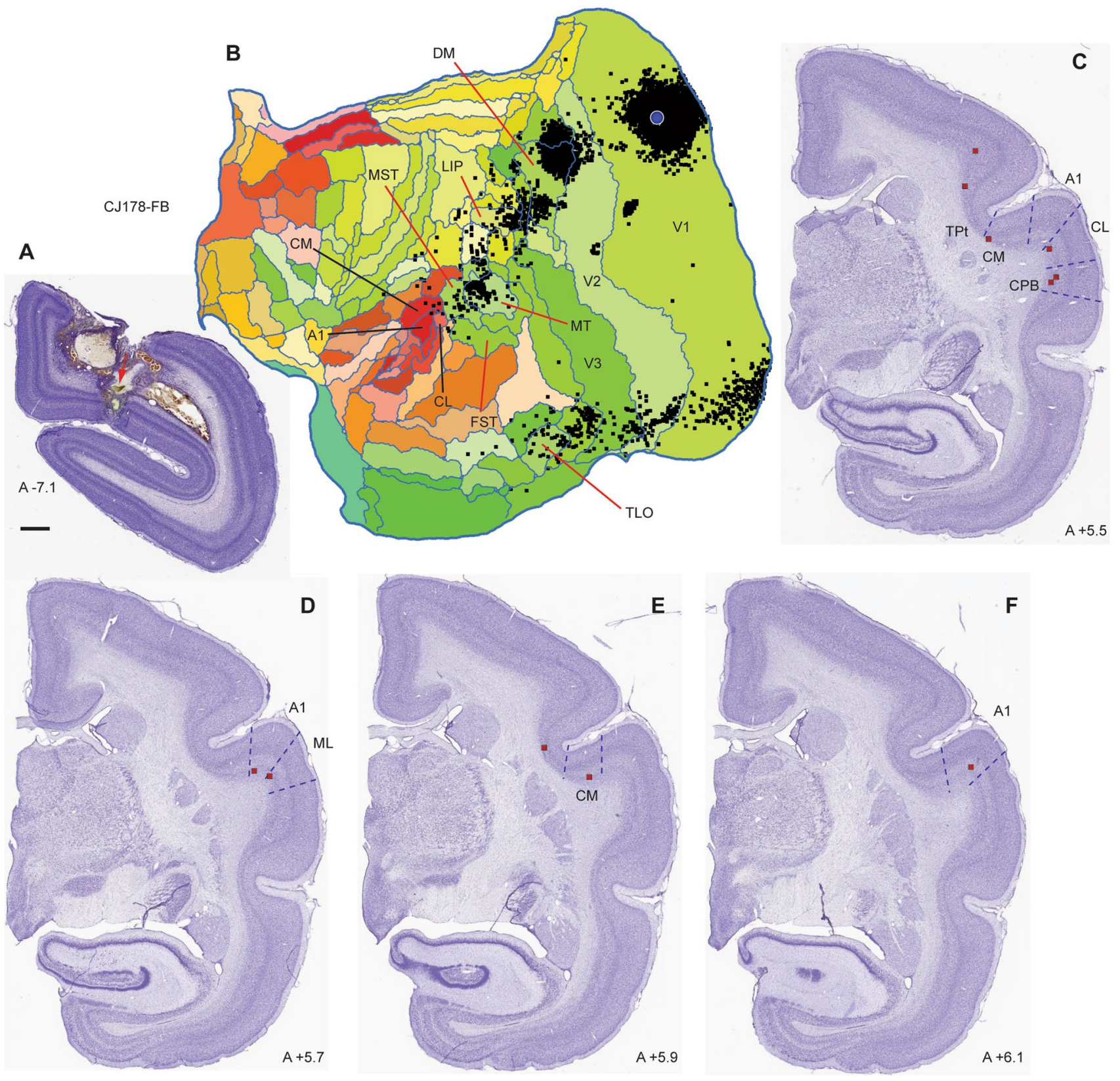

Fig. 4 Summary of results in case CJ178-FB. A Coronal section through the centre of the FB injection site. In this case there was damage to the cortex overlying the calcarine sulcus subsequent to the injection. The injection site is indicated by the red arrow. Scale bar: $1 \mathrm{~mm}$. B Unfolded reconstruction of the cortex showing the locations

possible involvement of the anterolateral belt area (AL), whereas in case CJ180-FB (Fig. 9C) it was centred in RT, with involvement of the rostrotemporal lateral area (RTL) and AL. Again, putative visual connections originated in the rostral parts of MST and FST, which corresponded to small proportions of the extrinsic label (0.96 and $1.09 \%$ in CJ122-DY and CJ180-FB, respectively). However, in these cases additional connections from putative visual association of neurons labelled by the tracer injection (black squares) following registration to the template. The injection site is indicated by the blue circle. C Examples of coronal sections containing labelled neurons in auditory cortex, with their locations indicated by red squares. Conventions as in Figs. 2 and 3

cortex in the inferior temporal cortex (areas TE1-TE3; see Fig. 9B, C).

Three injections were centred in the caudal belt areas. In case CJ64- FR (Fig. 9D), a small injection near the border of areas $\mathrm{CM}, \mathrm{CL}$ and $\mathrm{A} 1$ failed to label neurons in visual areas, despite clear long-range transport to the frontal lobe, and the presence of cross-modal projections from somatosensory cortex [second somatosensory (S2), parietal ventral 
Fig. 5 Results from case M820. A Location of the FB injection site in V1, visualised in a Nissl stained section. Scale bar: $1 \mathrm{~mm}$. B Pattern of label in the lateral geniculate nucleus, confirming placement in V1, away from the representation of the vertical meridian (White et al. 1998). Label resulting from an injection of anterograde tracer in V1 is also visible (green). Scale bar: $500 \mu \mathrm{m}$. C Example FB-labelled neurons (arrows) in auditory areas, visualised in digital images obtained at various magnifications (scale bars: $20 \mu \mathrm{m}$ ). The top left image shows a single cell in caudal parabelt cortex, the top right panel shows a small cluster of labelled neurons in area CL, and the bottom panel illustrates a line of layer 6 cells crossing the border between CL and CM

(PV) and granular insula (GI) areas]. Injections centred in the middle lateral area (ML), with variable involvement of adjacent areas (Table 1), were more heterogeneous in their results (Fig. 10A-C). The most rostral of these injections was a small FE deposit in case CJ64 (Fig. 10A), located right at the boundary between ML and the anterolateral area (AL), involving portions of both areas. In this case, no putative visual afferents were detected. The more caudal injections in ML (cases CJ180-CTBg and CJ802-CTBg; Fig. 10B, C) both resulted in label in area prostriata $(n=31$ and 183 neurons), which is a subdivision of the retrosplenial cortex where visual responses are routinely obtained (Yu et al. 2012), as well as variable amounts of label in areas such as the middle temporal area (MT), MST, FST, the representation of the far periphery of the visual field in V2, and the medial subdivision of area 19 adjacent to peripheral V2 (area 19M; Paxinos et al. 2012), which also predominantly represents peripheral vision (Rosa and Schmid 1995). Finally, an injection in the caudal parabelt area revealed sparse connections with MST, FST, and inferior temporal areas (Fig. 10D).

\section{Discussion}

The existence of polysensory responses in macaque cortex has been known for some time, particularly in regions of the superior temporal polysensory (STP) cortex, posterior parietal cortex, and caudal lateral sulcus, which are located at the interface of the classical visual, somatosensory and auditory cortices (Hyvärinen and Shelepin 1979; Bruce et al. 1981; Baylis et al. 1987; Hikosaka et al. 1988; Mazzoni et al. 1996; Schlack et al. 2005). This type of activity was initially thought to be dependent on bottom-up integration of unisensory processing pathways into specialised regions of the cortex, in light of a hierarchical processing model (Jones and Powell 1970; Felleman and Van Essen 1991). However, indications of auditory-induced modulations in the electrophysiological and haemodynamic activity of V1 (Giard and Peronnet 1999; Macaluso et al. 2000), followed by pioneering studies which revealed that $\mathrm{V} 1$ receives direct projections from both the STP cortex and auditory areas
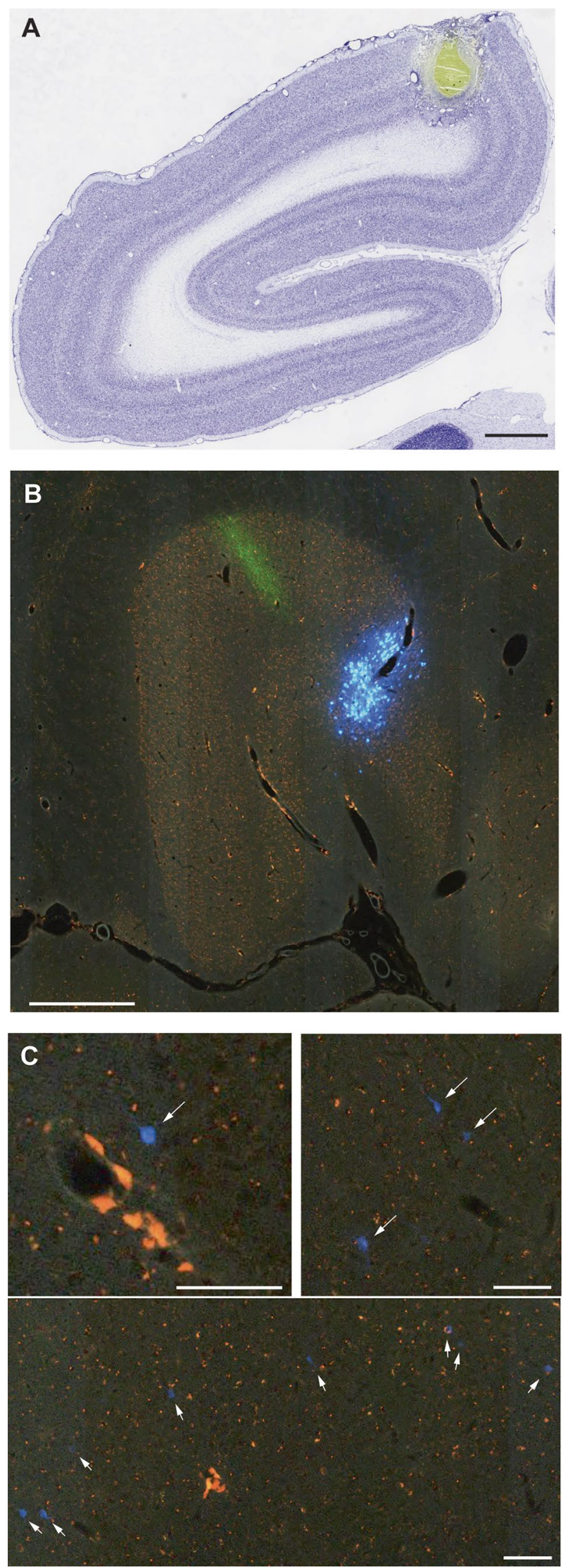
Fig. 6 Examples of the locations of labelled neurons in auditory and adjacent areas in case M820. Scale bar (top left): $1 \mathrm{~mm}$
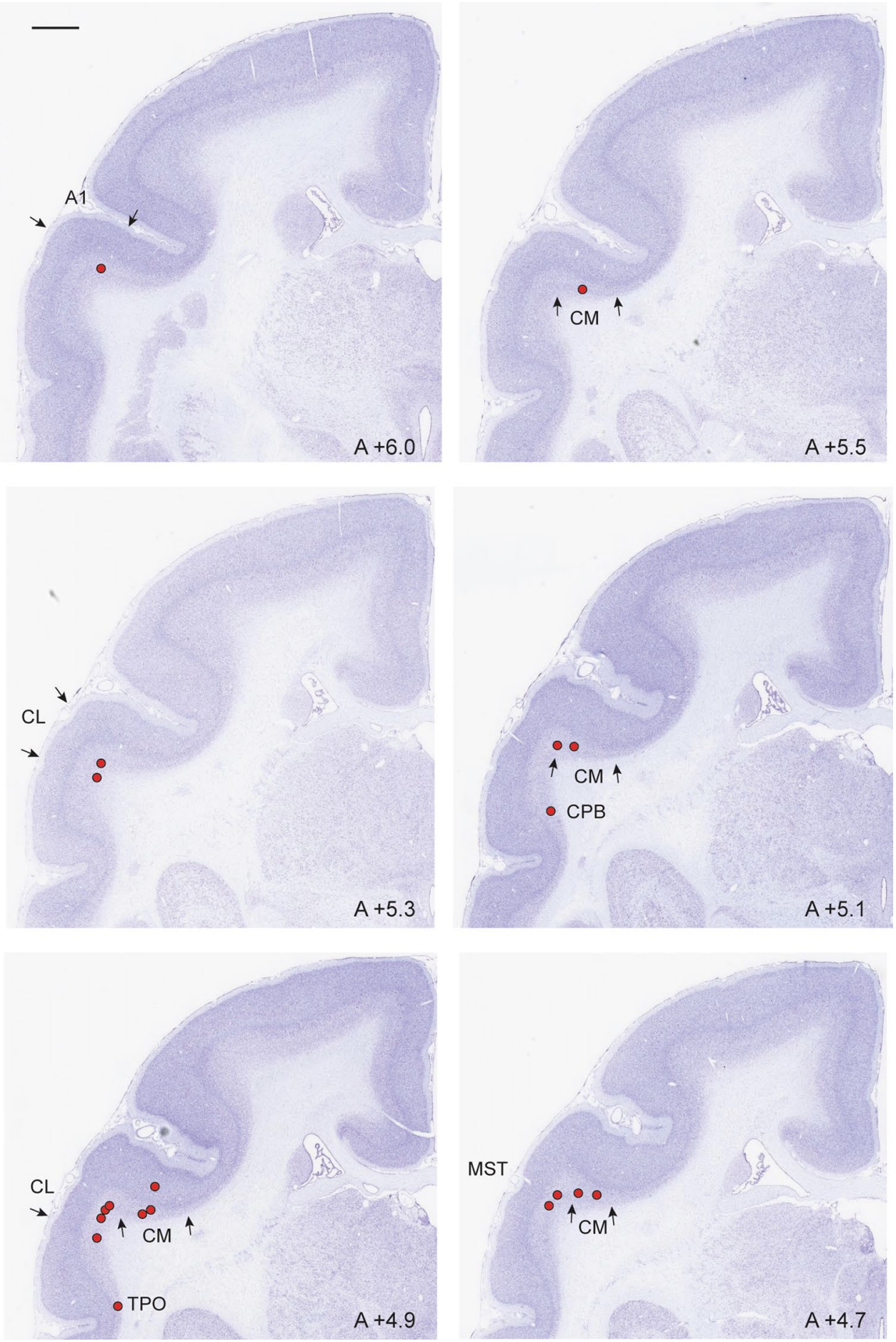

(Falchier et al. 2002; Rockland and Ojima 2003) have promoted a revision of multisensory integration models in the cortex. Here, we found evidence that cross-modal projections exist in the marmoset. As in the macaque, the projection originates primarily from the infragranular layers of caudal auditory areas. Although they form a small minority of the cortical extrinsic afferent projections to V1, auditory projections appear to be specific. For example, no labelled neurons are located in the rostral auditory cortex or other cortical areas located at similar or shorter distances from 


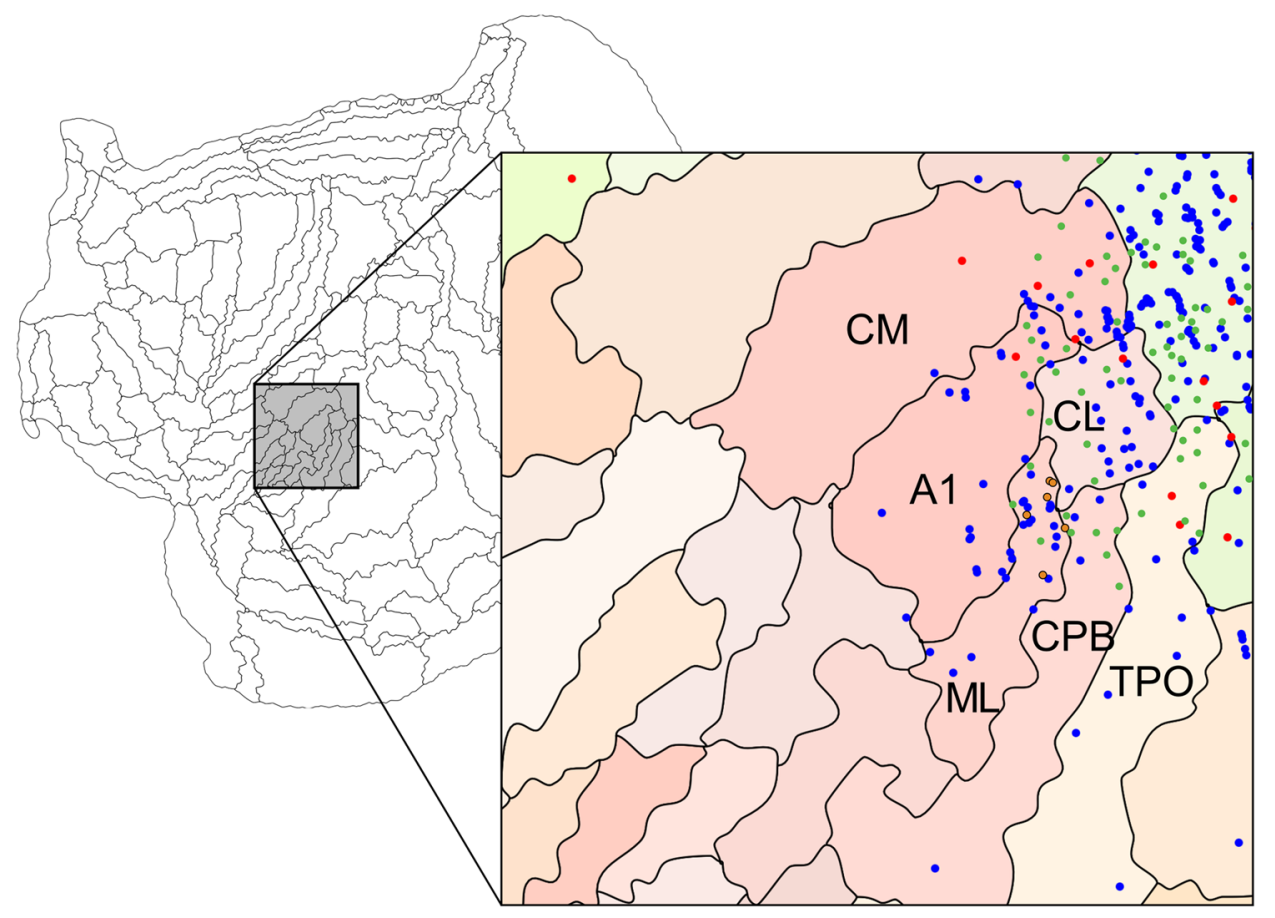

Fig. 7 Summary of the location of labelled neurons in auditory and adjacent areas, following V1 injections in 4 animals. The main panel is an expanded version of the caudal superior temporal cortex in the unfolded reconstruction of the cortex (magnified region is indicated in grey). The results obtained in animals CJ174 (blue circles) and CJ178 (red circles) were computationally registered to an unfolded reconstruction of the Paxinos et al. (2012) cortex template. Results

the injection sites. Moreover, in several of our cases labelled neurons were observed in the visual domains of the frontal lobe (areas $8 \mathrm{aV}$ and 45; Burman et al. 2006; Reser et al. 2013), demonstrating that the lack of other projections is not the result of short transport times following the injections. Future studies using high-sensitivity anterograde tracer injections in marmoset caudal auditory areas will be necessary to determine if the laminar targets of these projections are layers 1 and 6 of V1, as reported by Rockland and Ojima (2003) in the macaque brain.

The primary significance of these results is that they confirm that auditory areas send direct, monosynaptic connections to V1 in a New World monkey species. Like macaques, marmosets are simian primates, but, together with other New World monkeys, they have evolved in isolation from Old World monkeys, apes and humans for more than 40 million years (i.e. approximately half of the total evolutionary history of the Order Primates; Perelman et al. 2011; Rosa et al. 2018). This finding suggests that direct auditory influence on early visual processing originated early in primate evolution. Marmosets and macaques differ substantially in terms of body size, brain volume and degree of gyrification, and many behavioural characteristics (Stephan et al. 1981; from animals M820 (green) and M822 (orange points with black outlines) were approximately plotted onto the same reconstruction by a procedure involving identifying the location of neurons relative to histological borders (Nissl and myelin stains) in the native brains, and manually mapping these locations to the most similar sections in CJ174 or CJ178

Rosa et al. 2018). Thus, the preservation of similar auditory projections to V1 seems to suggest a general functional significance across different ecological niches.

There are a few differences in the results obtained in the two species, but these may be due in part to methodological issues. In general, the smaller size of the marmoset brain imposes the use of smaller volumes of tracer, in order to minimise the risk of contamination of adjacent areas or invasion of the white matter. Thus, the numbers of labelled cells in our experiments tend to be much lower than those reported by similar work in the macaque. Considering that the auditory projections to $\mathrm{V} 1$ form a relatively low percentage of the total afferent population $(<1.0 \%)$, the risk of false negatives is increased. In our study, all of the injections that revealed clear projections used the retrograde tracer $\mathrm{FB}$, which tends to form relatively large injection sites, and consequently label larger number of cells (particularly in comparison with dextran-based tracers such as FR and FE). FB was also used in most of the cases reported by Falchier et al. (2002).

In the macaque (Falchier et al. 2002), auditory projections to peripheral V1 appear to be more substantial than those to central vision. Although the sample is small, a 


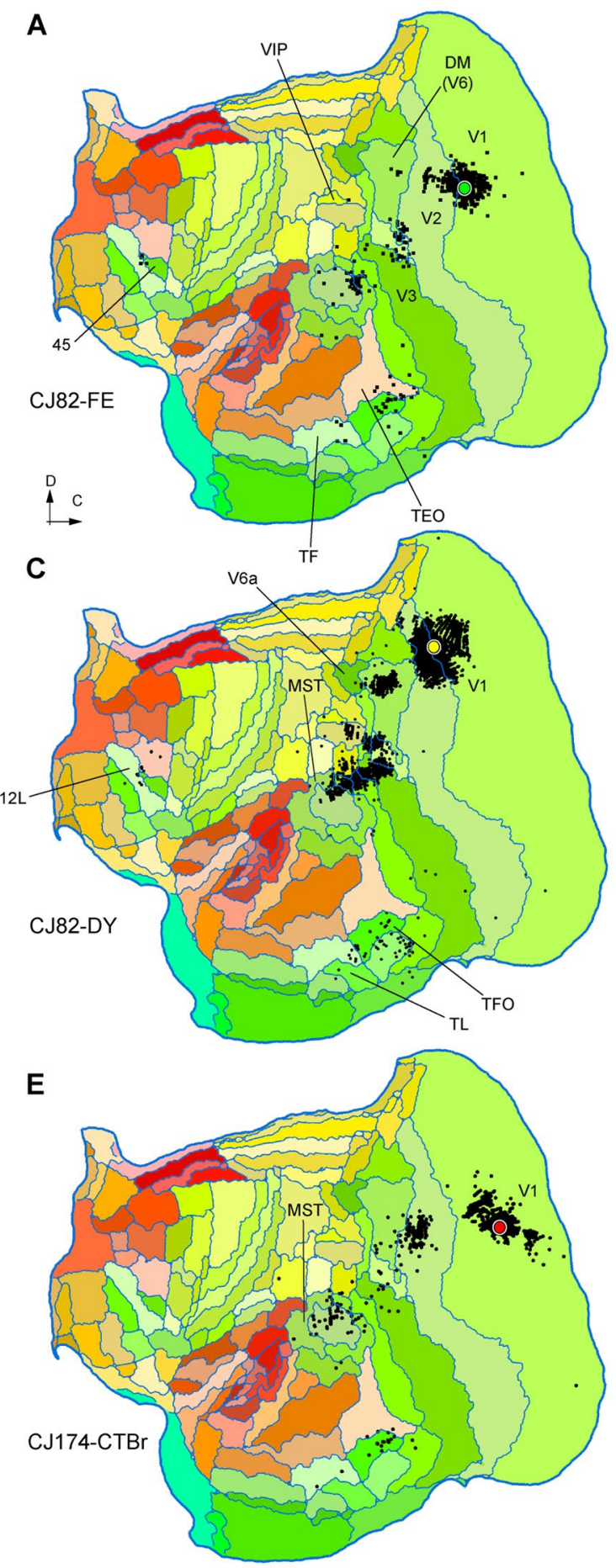

Fig. 8 A-E Unfolded reconstructions of the cortex showing the locations of labelled neurons following tracer injections which did not result in any labelled neurons in auditory cortex. The cases illustrated in $\mathbf{A}, \mathbf{B}, \mathbf{D}$ and $\mathbf{E}$ correspond to injections that were entirely contained

similar trend is also apparent in our materials, where the cases with injections in the calcarine sulcus (CJ174, CJ178) revealed higher percentages of labelled neurons in auditory cortex $(0.75 \%, 0.23 \%)$, in comparison with injections in the
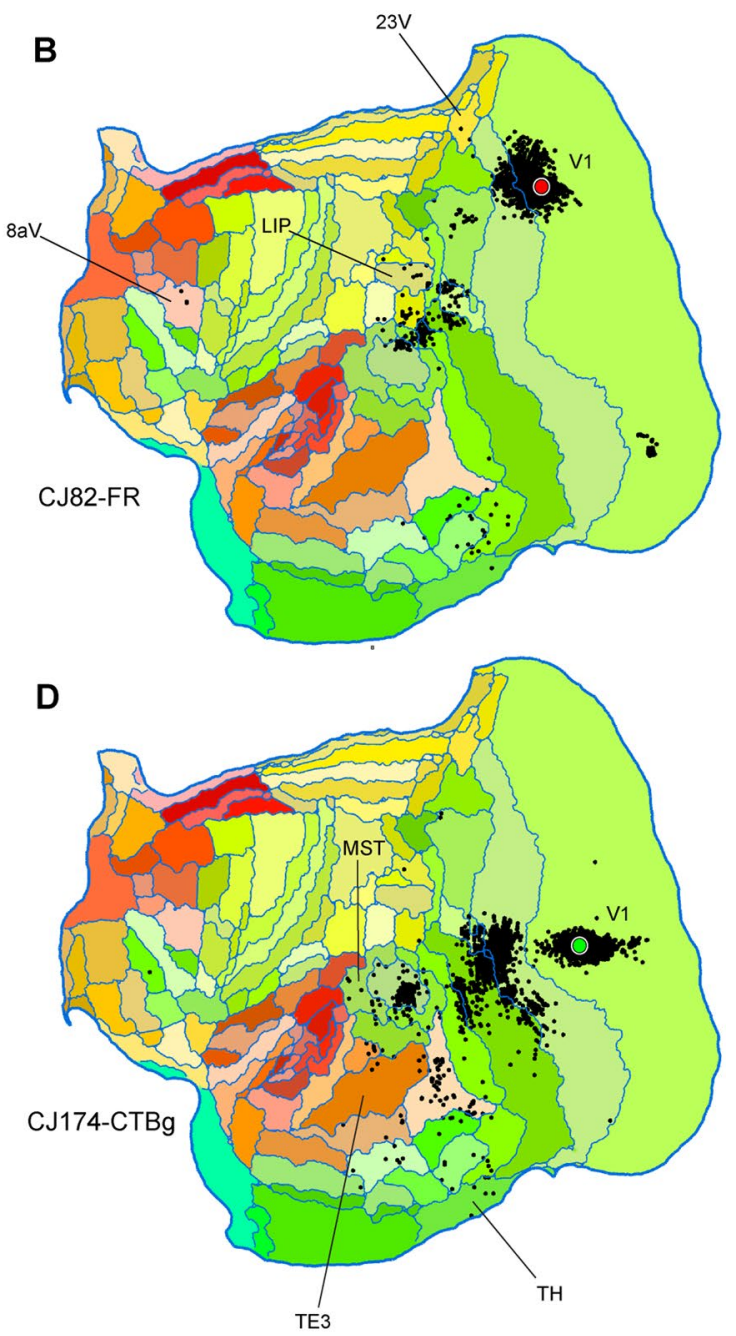

within V1, while the one illustrated in $\mathrm{C}$ was centred in V2, with slight V1 invasion. Conventions as in Fig. 2. Several cortical areas where long-range projection neurons were labelled are indicated for orientation. For abbreviations, see Table 2

parafoveal (M820) and foveal (M822) representations of V1 $(0.17 \%,<0.1 \%)$. No label in auditory cortex was detected in other cases, even though one of the animals (CJ82) had 2 injections (FR and DY) in the peripheral representation 

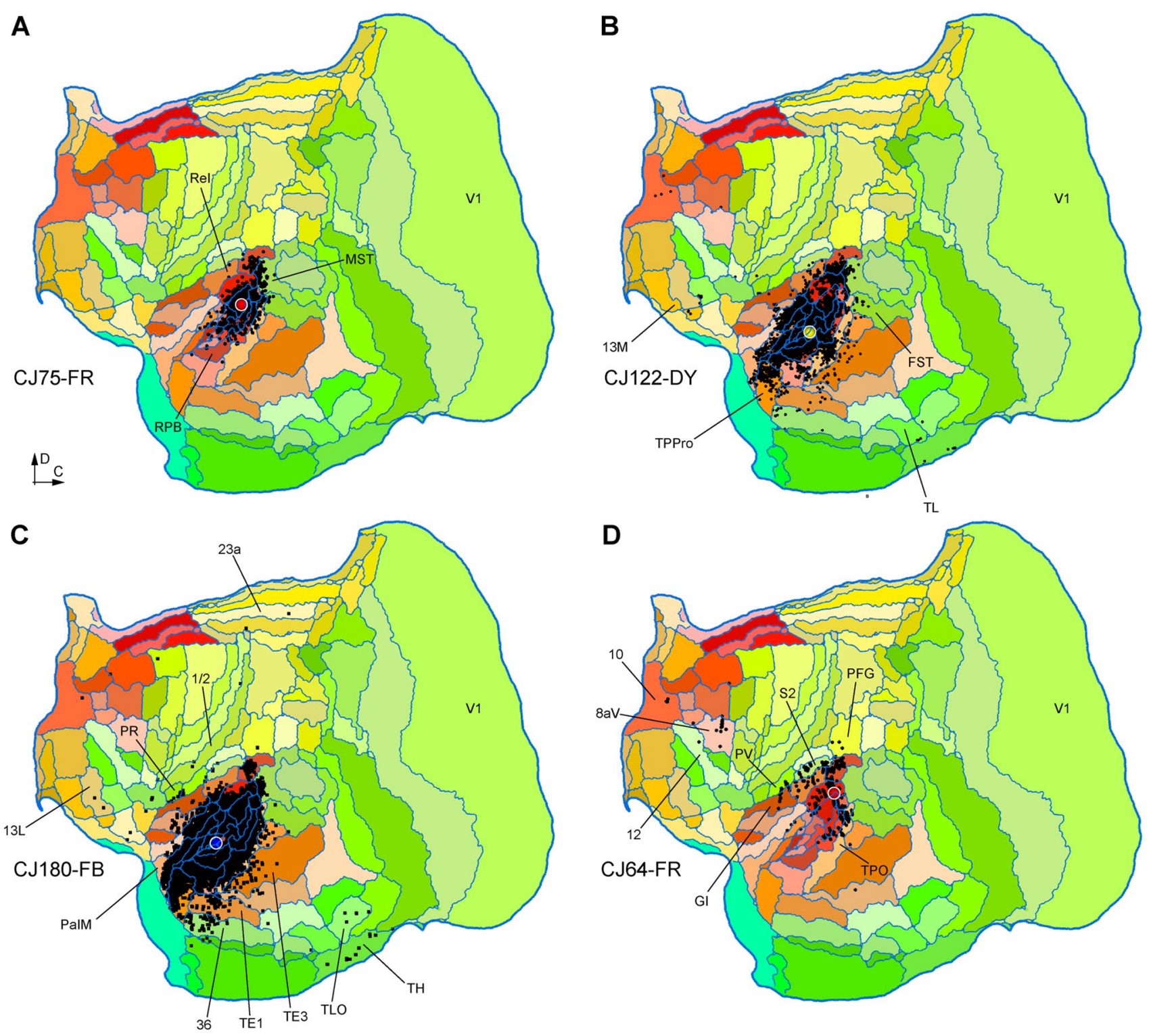

Fig. 9 A-D Unfolded reconstructions of the cortex showing the locations of labelled neurons in 4 injections in auditory cortex. The injections illustrated in panels $\mathbf{A}-\mathbf{C}$ were centred in auditory core areas (with the invasion of adjacent areas in $\mathbf{B}, \mathbf{C}$ ), while the one illustrated in $\mathbf{D}$ was centred in caudal belt area CM, with likely involvement of

(10-20 eccentricity), which resulted in substantial longrange transport. In considering these results, it may be relevant that the latter injections were very close to, or straddled the V1/ V2 border, where the vertical meridian of the visual field is represented. In humans, estimates of minimal detectable separation between auditory stimulus sources are highest when the stimuli are arranged vertically along the midline (Perrott and Saberi 1990), and the benefit of concurrent auditory stimulation in enhancing spatial localisation of visual stimuli is not observed near the midline (Perrott et al. 1993). This suggests the interpretation that auditory
A1. None of these injections resulted in retrogradely labelled neurons in V1. Several cortical areas where long-range projection neurons were labelled are indicated for orientation. For abbreviations, see Table 2

projections to V1 may be primarily directed to representations of portions of space away from the vertical meridian, where they can be of greatest value in stimulus localisation, rather than to the peripheral representation of this area in general. Other studies have shown that visual stimulus localisation is only enhanced by concurrent auditory stimulation in the peripheral visual field, which is usually defined as distance from the fovea along the horizontal dimension (Perrott et al. 1991; Frassinetti et al. 2002; Gleiss and Kayser 2013), and that the sensation of self-motion is enhanced by concurrent auditory and peripheral visual stimuli simulating 


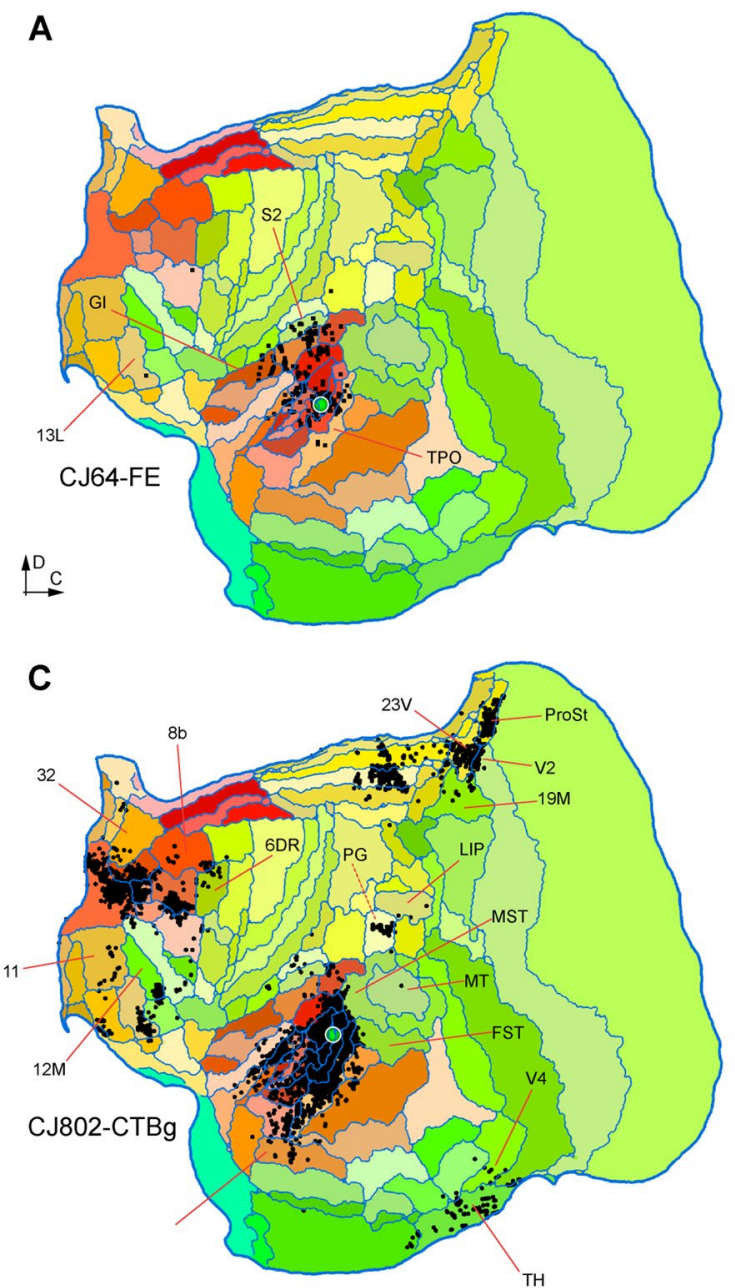

Fig. 10 A-D Unfolded reconstructions of the cortex showing the locations of labelled neurons in 4 injections in auditory cortex. A-C Are injections involving area ML, and D is an injection centred in the

vection (Keshavarz et al. 2014). There is also physiological evidence in macaque monkeys which indicates that concurrent auditory stimulation reduce the saccadic reaction times to peripheral visual stimuli (Wang et al. 2008). The faster conduction time of the auditory pathway may provide the opportunity of auditory processing to help in the localisation of visual stimuli away from the horizontal meridian, particularly in situations where the stimulus has low salience. For example, auditory responses in marmoset A1 and CM (two of the areas labelled in the present study) occur within 10-20 ms of the stimulus onset (Kajikawa et al. 2011; Rajan et al. 2013), in comparison with $>23 \mathrm{~ms}$ (median: $55 \mathrm{~ms}$ ) in the peripheral representation of V1 (Yu et al. 2012). Considering the relatively slow conduction of the visual pathway, and the fact that visual response latencies increase further when stimuli have low visibility (Bourne et al. 2002; Lui et al. 2012), a reciprocal projection from V1 to auditory areas would not provide a similar processing advantage. A
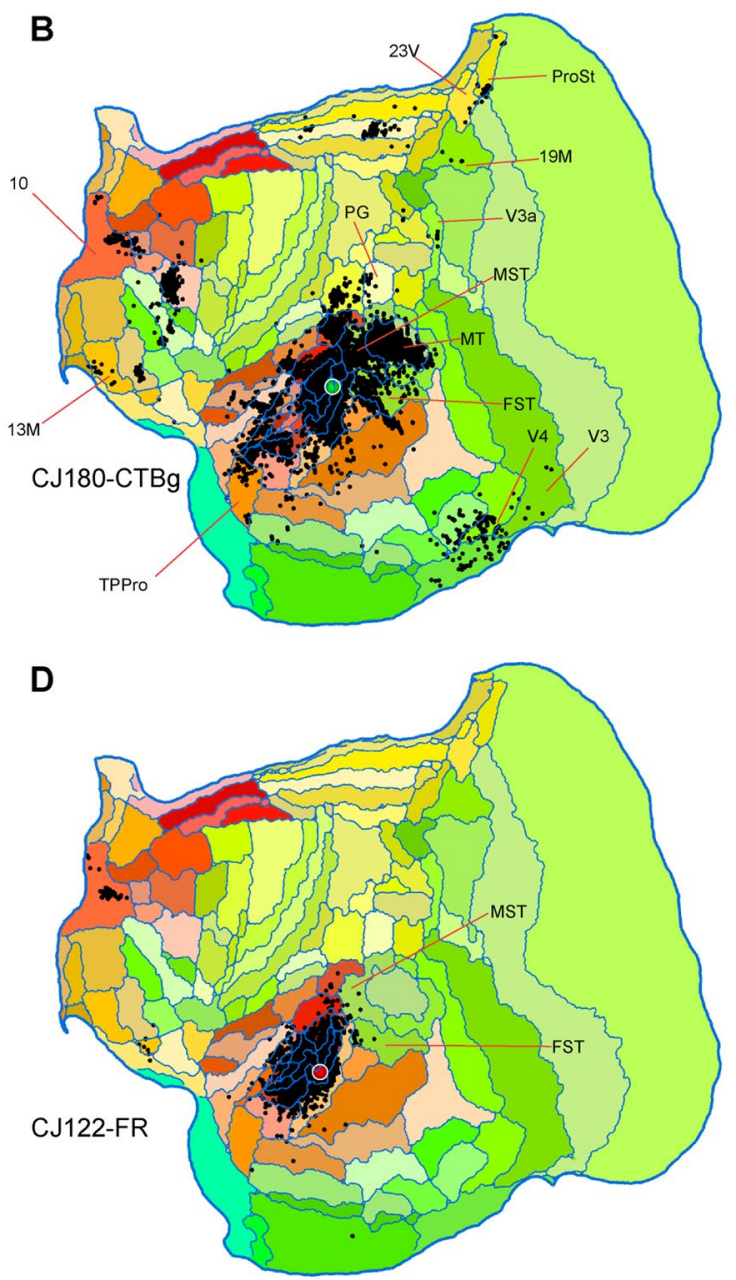

caudal parabelt. Several cortical areas where long-range projection neurons were labelled are indicated for orientation. For abbreviations, see Table 2

sparse projection from caudal auditory cortex to area MT (Palmer and Rosa 2006a) has been hypothesised to facilitate processing of visual motion, but recent single-neuron recording studies have not been able to confirm the existence of such an effect (Chaplin et al. 2018a, b).

Involvement of $\mathrm{V} 2$ in the injection sites does not seem to result in labelled neurons in auditory cortex. In addition to the 2 cases mentioned above, with injections near the representation of the vertical meridian, we had the opportunity to examine 8 additional injections that were entirely contained in $\mathrm{V} 2$, in regions representing eccentricities from foveal to far peripheral $\left(\sim 30^{\circ}\right)$, and at different distances from the V1 border. These data (not included in the present report, but freely available for perusal from http:// www.marmosetbrain.org) show no evidence of labelled neurons in any auditory areas. Thus, auditory projections do not target all early visual areas, suggesting that the physiological effects of concurrent auditory information 
in enhancing stimulus localisation occur at the earliest stage of visual processing, with areas downstream from V1 possibly sharing the physiological advantage conferred by audiovisual integration through information conveyed via V1.

We have also considered the possibility that the labelled neurons in auditory cortex were not projecting to $\mathrm{V} 1$, but were the result of the involvement of axons in the white matter subjacent to V1, traveling to some other visual area. We regard this as unlikely. At least one injection that resulted in auditory label was entirely contained within the cortex (M822-FB), and in only one of the others (CJ178-FB) the injection core invaded the white matter. Further, in the marmoset brain V1 is the only area found caudal to the anteroposterior level where the injections that labelled cells in auditory cortex were placed (note that in Figs. 2, 5 the entire section has the characteristic lamination of V1). Thus, the involvement of fibres of passage would require an unlikely scenario, whereby the auditory projection axons would reach towards the occipital pole, loop around the caudal end of the calcarine sulcus, and emerge rostrally to target another visual area.

Auditory projections to V1 have been well documented in cats (Hall and Lomber 2008), in a study which also found that the projections to the peripheral representation are more substantial. Neuroimaging and electroencephalographic studies have found various forms of modulation of the haemodynamic activity by concurrent audiovisual stimulation, and even evidence of auditory-evoked activity in visual areas (e.g. Giard and Peronnet 1999; Noesselt et al. 2007; Watkins et al. 2007; Brang et al. 2015; Azevedo et al. 2015; Petro et al. 2017), as well as evidence of functional connectivity between unimodal visual and auditory areas (Eckert et al. 2008). However, these modulations may not necessarily depend on monosynaptic connections (Chaplin et al. 2018a). In rodents, where the smaller brain and reduced number of areas imposes a different connectional architecture characterised by more direct interconnectivity between systems (Horvát et al. 2016; Gămănuţ et al. 2018), direct connections from auditory cortex to V1 appear to be more common (Larsen et al. 2009; Izraeli et al. 2002), but resemble those present in primates in terms of the infragranular predominance of the neurons forming connections (Charbonneau et al. 2012; Laramée et al. 2013). Unlike in primates, a reciprocal projection from $\mathrm{V} 1$ to auditory cortex has been described in prairie voles (Campi et al. 2010).

Using 8 tracer injections, we have explored the possibility of $\mathrm{V} 1$ projections to auditory areas. Two of these injections (CJ180- FB; Fig. 9C, and CJ122-DY; Fig. 9B) were relatively large, and involved subdivisions of the rostral core and belt areas. Neither of these resulted in any label in V1, or most other visual areas, despite revealing long-range connections from frontal and parahippocampal areas; thus, we are confident that the rostral auditory areas, which are thought to be involved in identification of sounds (Tian et al. 2001), do not participate in cross-modal connections with areas corresponding to low hierarchical levels of processing in visual cortex. One possible exception is area MST: in both cases, labelled neurons were found in the most rostral part of MST (as defined by myeloarchitecture; Palmer and Rosa 2006a, b). The spatial specificity of this connection could be seen as indication that MST, as currently defined in the marmoset, is heterogeneous. In particular, the marmoset superior temporal polysensory cortex (area TPO) could extend further dorsally than currently estimated, forming a narrow belt of cortex that separates caudal auditory areas CM and CL from MST proper (Fig. 11). Indeed, studies in the macaque have indicated that cytoarchitectural area TPO is heterogeneous (Padberg et al. 2003), and it is possible that a dorsal, more densely myelinated sector, has been confounded with MST in the parcellation proposed by Paxinos et al. (2012; see also Rosa et al. 1993). Evaluation of this hypothesis will require tracer injections in different portions of this region. In agreement with observations in the macaque (Hackett et al. 2007), we also found sparse somatosensory inputs to auditory areas, and many of the auditory injections labelled neurons in parahippocampal cortex, where sensory convergence has also been documented (Blatt et al. 2003).

The only clear cross-modal visual projections observed to auditory cortex in our data targeted auditory belt area ML. Both of the injections in this area (Fig. 10B, C) revealed a substantial projection from area prostriata, a subdivision of visual cortex characterised by large, peripheral receptive fields, and very short latencies to visual simulation (Yu et al. 2012). In the macaque, Falchier et al. (2010) also reported projections from prostriata to other subdivisions of caudal belt auditory cortex, suggesting that this projection targets multiple areas involved in auditory localisation. One of the injections in area ML labelled, in addition, substantial numbers of cells in area MT, and both injections resulted in scattered label in the peripheral representations of areas V2, V3 and V4. As a whole, the peripheral field specificity of these projections, together with the likely involvement of caudal auditory areas in spatial aspects of hearing (Tian et al. 2001) suggest that information from the far periphery of the visual field may modulate auditory processing, probably enhancing detection and localisation of stimuli.

In summary, our data add to the growing evidence pointing to the fact that, in addition to the bottom-up integration of sensory signals across hierarchical pathways converging into polysensory association areas, the primate brain contains sparse, but direct and specific pathways that allow information flow between areas traditionally conceived as unisensory. The auditory connections to V1 and MT (present results; Palmer and Rosa 2006a) are likely to play roles in enhancing stimulus detection and 
Paxinos et al. 2012

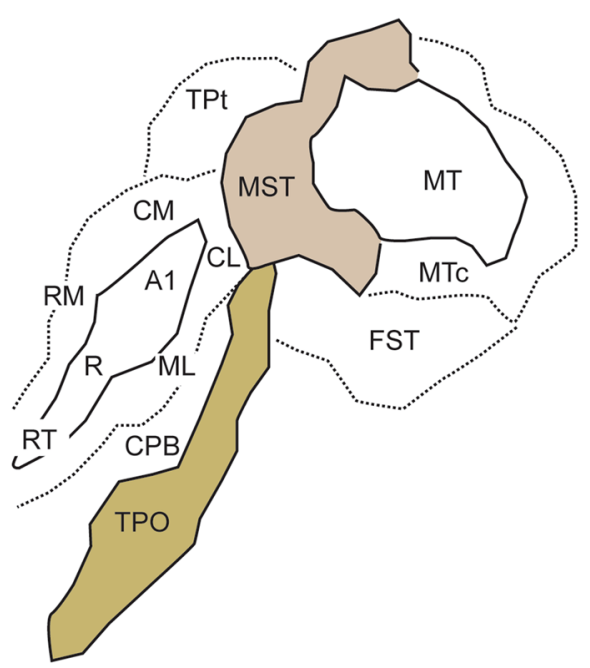

Fig. 11 A proposal for parcellation of marmoset area MST in two sectors, based on the present data and comparison with results obtained in the macaque monkey (Falchier et al. 2002). Left: original parcellation of the cortex near the tip of the superior temporal sulcus, according to the architectural study of Paxinos et al. (2012).

localisation, particularly away from the vertical meridian. Visual projections to auditory areas, primarily from prostriata and other peripheral representations, may provide a complementary role in detection, especially in situations when the signal to noise ratio of the auditory stimulus is low.

Funding Australian Research Council. Grant numbers: DP140101968, CE140100007. International Neuroinformatics Coordinating Facility Seed Funding Grant. Japan Agency for Medical Research and Development. Grant number: JP17dm0207001 (Brain/MINDS). Cod Spring Harbor Laboratory, Crick-Clay Professorship. Indian Institute of Technology Madras, H N Mahabala Chair.

\section{Compliance with ethical standards}

Ethical approval All procedures performed in studies using animals were in accordance with the ethical standards of the Institutions (Monash University and the RIKEN) at which the studies were conducted. All applicable international, national and institutional guidelines for the care and use of animals were followed.

Conflict of interest The authors declare that they have no conflict of interest.

Open Access This article is distributed under the terms of the Creative Commons Attribution 4.0 International License (http://creativeco mmons.org/licenses/by/4.0/), which permits unrestricted use, distribution, and reproduction in any medium, provided you give appropriate credit to the original author(s) and the source, provide a link to the Creative Commons license, and indicate if changes were made.
Revised

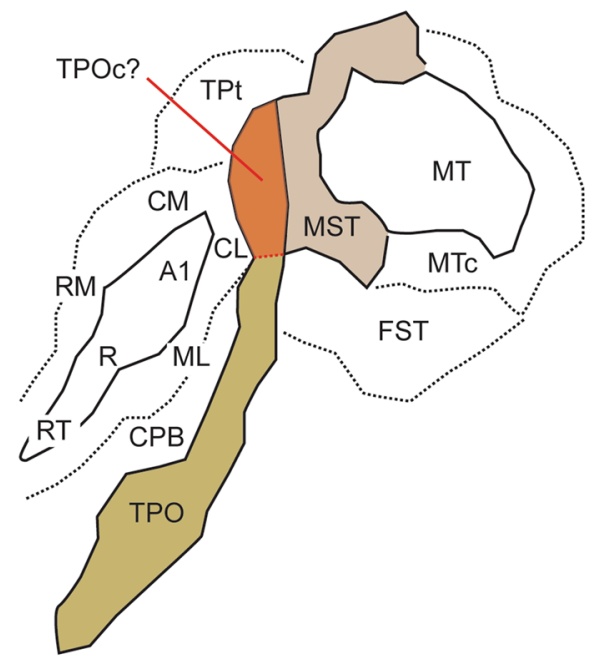

The diagram follows the orientation of Fig. 2. Right: The sector of MST where most labelled neurons are observed following auditory cortex injections could be a homologue of the caudal subdivision of area TPO (TPOc; Padberg et al. 2003), bringing in line the results obtained in macaque and marmoset monkeys

\section{References}

Alsius A, Navarra J, Campbell R, Soto-Faraco S (2005) Audiovisual integration of speech falters under high attention demands. Curr Biol 15:839-843

Angelucci A, Clasca F, Sur M (1996) Anterograde axonal tracing with the subunit B of cholera toxin: a highly sensitive immunohistochemical protocol for revealing fine axonal morphology in adult and neonatal brains. J Neurosci Methods 65:101-112

Azevedo FA, Ortiz-Rios M, Li Q, Logothetis NK, Keliris GA (2015) A potential role of auditory induced modulations in primary visual cortex. Multisens Res 28:331-349

Battaglia PW, Jacobs RA, Aslin RN (2003) Bayesian integration of visual and auditory signals for spatial localization. J Opt Soc Am A 20:1391-1397

Baylis GC, Rolls ET, Leonard CM (1987) Functional subdivisions of the temporal lobe neocortex. J Neurosci 7:330-342

Beauchamp MS (2005) See me, hear me, touch me: multisensory integration in lateral occipital-temporal cortex. Curr Opin Neurobiol 15:145-153

Bendor D, Wang X (2008) Neural response properties of primary, rostral, and rostrotemporal core fields in the auditory cortex of marmoset monkeys. J Neurophysiol 100:888-906

Benevento LA, Fallon J, Davis BJ, Rezak M (1977) Auditory-visual interaction in single cells in the cortex of the superior temporal sulcus and the orbital frontal cortex of the macaque monkey. Exp Neurol 57:849-872

Blatt GJ, Pandya DN, Rosene DL (2003) Parcellation of cortical afferents to three distinct sectors in the parahippocampal gyrus of the rhesus monkey: an anatomical and neurophysiological study. J Comp Neurol 466:161-179

Bourne JA, Tweedale R, Rosa MGP (2002) Physiological responses of New World monkey V1 neurons to stimuli defined by coherent motion. Cereb Cortex 12:1132-1145 
Brang D, Towle VL, Suzuki S, Hillyard SA, Di Tusa S, Dai Z, Tao J, Wu S, Grabowecky M (2015) Peripheral sounds rapidly activate visual cortex: evidence from electrocorticography. J Neurophysiol 114:3023-3028

Bruce C, Desimone R, Gross CG (1981) Visual properties of neurons in a polysensory area in superior temporal sulcus of the macaque. J Neurophysiol 46:369-384

Burman KJ, Rosa MGP (2009) Architectural subdivisions of medial and orbital frontal cortices in the marmoset monkey (Callithrix jacchus). J Comp Neurol 514:11-29

Burman KJ, Palmer SM, Gamberini M, Rosa MGP (2006) Cytoarchitectonic subdivisions of the dorsolateral frontal cortex of the marmoset monkey (Callithrix jacchus), and their projections to dorsal visual areas. J Comp Neurol 495:149-172

Burman KJ, Reser DH, Yu H-H, Rosa MGP (2010) Cortical input to the frontal pole of the marmoset monkey. Cereb Cortex 21:1712-1737

Burman KJ, Bakola S, Richardson KE, Reser DH, Rosa MGP (2014a) Patterns of cortical input to the primary motor cortex in the marmoset monkey. J Comp Neurol 522:811-843

Burman KJ, Bakola S, Richardson KE, Reser DH, Rosa MGP (2014b) Patterns of afferent input to the caudal and rostral areas of the dorsal premotor cortex (6DC and 6DR) in the marmoset monkey. J Comp Neurol 522:3683-3716

Burman KJ, Bakola S, Richardson KE, Yu H-H, Reser DH, Rosa MGP (2015) Cortical and thalamic projections to cytoarchitectural areas $6 \mathrm{Va}$ and $8 \mathrm{C}$ of the marmoset monkey: connectionally distinct subdivisions of the lateral premotor cortex. $\mathrm{J}$ Comp Neurol 523:1222-1247

Burr D, Alais D (2006) Combining visual and auditory information. Prog Brain Res 155:243-258

Campi KL, Bales KL, Grunewald R, Krubitzer L (2010) Connections of auditory and visual cortex in the prairie vole (Microtus ochrogaster): evidence for multisensory processing in primary sensory areas. Cereb Cortex 20:89-108

Cappe C, Barone P (2005) Heteromodal connections supporting multisensory integration at low levels of cortical processing in the monkey. Eur J Neurosci 22:2886-2902

Chaplin TA, Yu H-H, Rosa MGP (2013) Representation of the visual field in the primary visual area of the marmoset monkey: magnification factors, point-image size, and proportionality to retinal ganglion cell density. J Comp Neurol 521:1001-1019

Chaplin TA, Allitt BJ, Hagan MA, Rosa MGP, Rajan R, Lui LL (2018a) Auditory motion does not modulate spiking activity in the middle temporal and medial superior temporal visual areas. Eur J Neurosci 48:2013-2029

Chaplin TA, Rosa MGP, Lui LL (2018b) Auditory and visual motion processing and integration in the primate cerebral cortex. Front Neural Circuits (in press)

Charbonneau V, Laramee ME, Boucher V, Bronchti G, Boire D (2012) Cortical and subcortical projections to primary visual cortex in anophthalmic, enucleated and sighted mice. Eur J Neurosci 36:2949-2963

Condé F (1987) Further studies on the use of the fluorescent tracers fast blue and diamidino yellow: effective uptake area and cellular storage sites. J Neurosci Methods 21:31-43

Eckert MA, Kamdar NV, Chang CE, Beckmann CF, Greicius MD, Menon V (2008) A cross-modal system linking primary auditory and visual cortices: evidence from intrinsic fMRI connectivity analysis. Hum Brain Mapp 29:848-857

Eliades SJ, Miller CT (2017) Marmoset vocal communication: behavior and neurobiology. Dev Neurobiol 77:286-299

Falchier A, Clavagnier S, Barone P, Kennedy H (2002) Anatomical evidence of multimodal integration in primate striate cortex. $\mathrm{J}$ Neurosci 22:5749-5759
Falchier A, Schroeder CE, Hackett TA, Lakatos P, Nascimento-Silva S, Ulbert I, Karmos G, Smiley JF (2010) Projection from visual areas $\mathrm{V} 2$ and prostriata to caudal auditory cortex in the monkey. Cereb Cortex 20:1529-1538

Felleman DJ, Van Essen DC (1991) Distributed hierarchical processing in the primate cerebral cortex. Cereb Cortex 1:1-47

Frassinetti F, Bolognini N, Ladavas E (2002) Enhancement of visual perception by crossmodal visuo-auditory interaction. Exp Brain Res 147:332-343

Fritsches KA, Rosa MGP (1996) Visuotopic organisation of striate cortex in the marmoset monkey (Callithrix jacchus). J Comp Neurol 372:264-282

Gallyas F (1979) Silver staining of myelin by means of physical development. Neurol Res 1:203-209

Gămănuț R, Kennedy H, Toroczkai Z, Ercsey-Ravasz M, Van Essen DC, Knoblauch K, Burkhalter A (2018) The mouse cortical connectome, characterized by an ultra-dense cortical graph, maintains specificity by distinct connectivity profiles. Neuron 97:698-715 e610

Ghazanfar AA, Schroeder CE (2006) Is neocortex essentially multisensory? Trends Cogn Sci 10:278-285

Giard MH, Peronnet F (1999) Auditory-visual integration during multimodal object recognition in humans: a behavioral and electrophysiological study. J Cogn Neurosci 11:473-490

Gleiss S, Kayser C (2013) Eccentricity dependent auditory enhancement of visual stimulus detection but not discrimination. Front Integr Neurosci 7(52):1-8

Hackett TA, Smiley JF, Ulbert I, Karmos G, Lakatos P, de la Mothe LA, Schroeder CE (2007) Sources of somatosensory input to the caudal belt areas of auditory cortex. Perception 36:1419-1430

Hagan MA, Rosa MGP, Lui LL (2017) Neural plasticity following lesions of the primate occipital lobe: The marmoset as an animal model for studies of blindsight. Dev Neurobiol 77:314-327

Hall AJ, Lomber SG (2008) Auditory cortex projections target the peripheral field representation of primary visual cortex. Exp Brain Res 190:413-430

Hikosaka K, Iwai E, Saito H, Tanaka K (1988) Polysensory properties of neurons in the anterior bank of the caudal superior temporal sulcus of the macaque monkey. J Neurophysiol 60:1615-1637

Horvat S, Gamanut R, Ercsey-Ravasz M, Magrou L, Gamanut B, Van Essen DC, Burkhalter A, Knoblauch K, Toroczkai Z, Kennedy $\mathrm{H}$ (2016) Spatial embedding and wiring cost constrain the functional layout of the cortical network of rodents and primates. PLoS Biol 14(7):e1002512:1-30

Hyvärinen J, Shelepin Y (1979) Distribution of visual and somatic functions in the parietal associative area 7 of the monkey. Brain Res 169:561-564

Izraeli R, Koay G, Lamish M, Heicklen-Klein AJ, Heffner HE, Heffner RS, Wollberg Z (2002) Cross-modal neuroplasticity in neonatally enucleated hamsters: structure, electrophysiology and behaviour. Eur J Neurosci 15:693-712

Jones EG, Powell TP (1970) An anatomical study of converging sensory pathways within the cerebral cortex of the monkey. Brain 93:793-820

Kajikawa Y, Camalier CR, de la Mothe LA, D'Angelo WR, SterbingD'Angelo SJ, Hackett TA (2011) Auditory cortical tuning to band-pass noise in primate $\mathrm{A} 1$ and $\mathrm{CM}$ : a comparison to pure tones. Neurosci Res 70:401-407

Kayser C, Petkov CI, Logothetis NK (2009) Multisensory interactions in primate auditory cortex: fMRI and electrophysiology. Hear Res 258:80-88

Keizer K, Kuypers HG, Huisman AM, Dann O (1983) Diamidino yellow dihydrochloride (DY. $2 \mathrm{HCl}$ ); a new fluorescent retrograde neuronal tracer, which migrates only very slowly out of the cell. Exp Brain Res 51:179-191 
Keshavarz B, Hettinger LJ, Vena D, Campos JL (2014) Combined effects of auditory and visual cues on the perception of vection. Exp Brain Res 232:827-836

Laramée ME, Rockland KS, Prince S, Bronchti G, Boire D (2013) Principal component and cluster analysis of layer $\mathrm{V}$ pyramidal cells in visual and non-visual cortical areas projecting to the primary visual cortex of the mouse. Cereb Cortex 23:714-728

Larsen DD, Luu JD, Burns ME, Krubitzer L (2009) What are the effects of severe visual impairment on the cortical organization and connectivity of primary visual cortex? Front Neuroanat 3(30):1-16

Lin MK, Takahashi YS, Huo B-X, Hanada M, Nagashima J, Hata J, Tolpygo AS, Ram K, Lee BC, Miller MI, Rosa MGP, Sasaki E, Iriki A, Okano H, Mitra PP (2018) A high-throughput neurohistological pipeline for brain-wide mesoscale connectivity mapping of the common marmoset. bioRxiv 315804 (preprint in open access repository)

Lui LL, Dobiecki AE, Bourne JA, Rosa MGP (2012) Breaking camouflage: responses of neurons in the middle temporal area to stimuli defined by coherent motion. Eur J Neurosci 36:2063-2076

Lyon DC, Kaas JH (2001) Connectional and architectonic evidence for dorsal and ventral V3, and dorsomedial area in marmoset monkeys. J Neurosci 21:249-261

Macaluso E, Driver J (2005) Multisensory spatial interactions: a window onto functional integration in the human brain. Trends Neurosci 28:264-271

Macaluso E, Frith CD, Driver J (2000) Modulation of human visual cortex by crossmodal spatial attention. Science 289:1206-1208

Majka P, Chaplin TA, Yu H-H, Tolpygo A, Mitra PP, Wojcik DK, Rosa MGP (2016) Towards a comprehensive atlas of cortical connections in a primate brain: Mapping tracer injection studies of the common marmoset into a reference digital template. J Comp Neurol 524:2161-2181

Mazzoni P, Bracewell RM, Barash S, Andersen RA (1996) Spatially tuned auditory responses in area LIP of macaques performing delayed memory saccades to acoustic targets. J Neurophysiol 75:1233-1241

Miller CT, Freiwald WA, Leopold DA, Mitchell JF, Silva AC, Wang $X$ (2016) Marmosets: a neuroscientific model of human social behavior. Neuron 90:219-233

Mitchell JF, Leopold DA (2015) The marmoset monkey as a model for visual neuroscience. Neurosci Res 93:20-46

Noesselt T, Rieger JW, Schoenfeld MA, Kanowski M, Hinrichs H, Heinze HJ, Driver J (2007) Audiovisual temporal correspondence modulates human multisensory superior temporal sulcus plus primary sensory cortices. J Neurosci 27:11431-11441

Oikonomidis L, Santangelo AM, Shiba Y, Clarke FH, Robbins TW, Roberts AC (2017) A dimensional approach to modeling symptoms of neuropsychiatric disorders in the marmoset monkey. Dev Neurobiol 77:328-353

Okano H, Kishi N (2017) Investigation of brain science and neurologi$\mathrm{cal} /$ psychiatric disorders using genetically modified non-human primates. Curr Opin Neurobiol 50:1-6

Okano H, Sasaki E, Yamamori T, Iriki A, Shimogori T, Yamaguchi Y, Kasai K, Miyawaki A (2016) Brain/MINDS: A Japanese national brain project for marmoset neuroscience. Neuron 2016 92:582-590

Padberg J, Seltzer B, Cusick CG (2003) Architectonics and cortical connections of the upper bank of the superior temporal sulcus in the rhesus monkey: an analysis in the tangential plane. J Comp Neurol 467:418-434

Palmer SM, Rosa MGP (2006a) Quantitative analysis of the corticocortical projections to the middle temporal area in the marmoset monkey: evolutionary and functional implications. Cereb Cortex 16:1361-1375
Palmer SM, Rosa MGP (2006b) A distinct anatomical network of cortical areas for analysis of motion in far peripheral vision. Eur $\mathbf{J}$ Neurosci 24:2389-2405

Paxinos G, Watson C, Petrides M, Rosa MGP, Tokuno H (2012) The marmoset brain in stereotaxic coordinates, 1 st ed. Academic Press, Cambridge

Perelman P, Johnson WE, Roos C, Seuanez HN, Horvath JE, Moreira MA, Kessing B, Pontius J, Roelke M, Rumpler Y, Schneider MP, Silva A, O'Brien SJ, Pecon-Slattery J (2011) A molecular phylogeny of living primates. PLoS Genet 7(3):e1001342

Perrott DR, Saberi K (1990) Minimum audible angle thresholds for sources varying in both elevation and azimuth. J Acoust Soc Am 87:1728-1731

Perrott DR, Sadralodabai T, Saberi K, Strybel TZ (1991) Aurally aided visual search in the central visual field: effects of visual load and visual enhancement of the target. Hum Factors 33:389-400

Perrott DR, Costantino B, Cisneros J (1993) Auditory and visual localization performance in a sequential discrimination task. J Acoust Soc Am 93:2134-2138

Petro LS, Paton AT, Muckli L (2017) Contextual modulation of primary visual cortex by auditory signals. Philos Trans R Soc Lond B Biol Sci 372(1714):20160104:1-9

Pinskiy V, Jones J, Tolpygo AS, Franciotti N, Weber K, Mitra PP (2015) High-throughput method of whole-brain sectioning, using the tape-transfer technique. PLoS One 10(7):e0102363

Rajan R, Dubaj V, Reser DH, Rosa MGP (2013) Auditory cortex of the marmoset monkey-complex responses to tones and vocalizations under opiate anaesthesia in core and belt areas. Eur $\mathbf{J}$ Neurosci 37:924-941

Reser DH, Burman KJ, Yu H-H, Chaplin TA, Richardson KE, Worthy KH, Rosa MGP (2013) Contrasting patterns of cortical input to architectural subdivisions of the area 8 complex: a retrograde tracing study in marmoset monkeys. Cereb Cortex 23:1901-1922

Rockland KS, Ojima H (2003) Multisensory convergence in calcarine visual areas in macaque monkey. Int J Psychophysiol 50:19-26

Rosa MGP, Schmid LM (1995) Visual areas in the dorsal and medial extrastriate cortices of the marmoset. J Comp Neurol 359:272-299

Rosa MGP, Tweedale R (2000) Visual areas in lateral and ventral extrastriate cortices of the marmoset monkey. J Comp Neurol 422:621-651

Rosa MGP, Soares JG, Fiorani M Jr, Gattass R (1993) Cortical afferents of visual area MT in the Cebus monkey: possible homologies between new and old world monkeys. Vis Neurosci 10:827-855

Rosa MGP, Palmer SM, Gamberini M, Tweedale R, Pinon MC, Bourne JA (2005) Resolving the organization of the New World monkey third visual complex: the dorsal extrastriate cortex of the marmoset (Callithrix jacchus). J Comp Neurol 483:164-191

Rosa MGP, Palmer SM, Gamberini M, Burman KJ, Yu H-H, Reser DH, Bourne JA, Tweedale R, Galletti C (2009) Connections of the dorsomedial visual area: pathways for early integration of dorsal and ventral streams in extrastriate cortex. J Neurosci 29:4548-4563

Rosa MGP, Soares JGM, Chaplin TA, Majka P, Bakola S, Phillips KA, Reser DH, Gattass R (2018) Cortical afferents of area 10 in Cebus monkeys: implications for the evolution of the frontal pole. Cereb Cortex, in press (epub ahead of print)

Ross LA, Saint-Amour D, Leavitt VM, Javitt DC, Foxe JJ (2006) Do you see what I am saying? Exploring visual enhancement of speech comprehension in noisy environments. Cereb Cortex 17:1147

Schlack A, Sterbing-D’Angelo SJ, Hartung K, Hoffmann KP, Bremmer F (2005) Multisensory space representations in the macaque ventral intraparietal area. J Neurosci 25:4616-4625

Schmued L, Kyriakidis K, Heimer L (1990) In vivo anterograde and retrograde axonal transport of the fluorescent 
rhodamine-dextran-amine, Fluoro-Ruby, within the CNS. Brain Res 526:127-134

Schroeder CE, Smiley J, Fu KG, McGinnis T, O’Connell MN, Hackett TA (2003) Anatomical mechanisms and functional implications of multisensory convergence in early cortical processing. Int $\mathbf{J}$ Psychophysiol 50:5-17

Silva AC (2017) Anatomical and functional neuroimaging in awake, behaving marmosets. Dev Neurobiol 77:373-389

Smiley JF, Falchier A (2009) Multisensory connections of monkey auditory cerebral cortex. Hear Res 258:37-46

Smiley JF, Hackett TA, Ulbert I, Karmas G, Lakatos P, Javitt DC, Schroeder CE (2007) Multisensory convergence in auditory cortex, I. Cortical connections of the caudal superior temporal plane in macaque monkeys. J Comp Neurol 502:894-923

Solomon SG, Rosa MGP (2014) A simpler primate brain: the visual system of the marmoset monkey. Front Neural Circuits 8(96): $1-24$

Stephan H, Frahm H, Baron G (1981) New and revised data on volumes of brain structures in insectivores and primates. Folia Primatol 35:1-29

Tian B, Reser DH, Durham A, Kustov A, Rauschecker JP (2001) Functional specialization in rhesus monkey auditory cortex. Science 292:290-293 van Atteveldt N, Murray MM, Thut G, Schroeder CE (2014) Multisensory integration: flexible use of general operations. Neuron 81:1240-1253

Wang Y, Celebrini S, Trotter Y, Barone P (2008) Visuo-auditory interactions in the primary visual cortex of the behaving monkey: electrophysiological evidence. BMC Neurosci 9(79):1-15

Watkins S, Shams L, Josephs O, Rees G (2007) Activity in human V1 follows multisensory perception. NeuroImage 37:572-578

White AJ, Wilder HD, Goodchild AK, Sefton AJ, Martin PR (1998) Segregation of receptive field properties in the lateral geniculate nucleus of a New-World monkey, the marmoset Callithrix jacchus. J Neurophysiol 80:2063-2076

Witten IB, Knudsen EI (2005) Why seeing is believing: merging auditory and visual worlds. Neuron 48:489-496

Wong-Riley M (1979) Changes in the visual system of monocularly sutured or enucleated cats demonstrable with cytochrome oxidase histochemistry. Brain Res 171:11-28

Yau JM, DeAngelis GC, Angelaki DE (2015) Dissecting neural circuits for multisensory integration and crossmodal processing. Philos Trans R Soc Lond B Biol Sci 370(1677):20140203:1-15

Yu H-H, Chaplin TA, Davies AJ, Verma R, Rosa MGP (2012) A specialized area in limbic cortex for fast analysis of peripheral vision. Curr Biol 22:1351-1357 